\title{
Dynamical behavior and exact solution in invariant manifold for a septic derivative nonlinear Schrödinger equation
}

\author{
Temesgen Desta Leta • Jibin Li
}

Received: 7 December 2016 / Accepted: 4 March 2017 / Published online: 17 March 2017

(C) The Author(s) 2017. This article is an open access publication

\begin{abstract}
In this paper, we consider a pulse dynamics in nonlinear optics (fiber-optic communications) in the presence of both self-steepening and septic nonlinear effects. Propagating profiles of the septic derivative nonlinear Schrödinger model which are isolated via coupled integrable invariants of motion, that admits exact solution, are investigated by a method of dynamical systems. By investigating the dynamical behavior and bifurcation of phase portraits of the traveling wave system, we obtain possible explicit exact parametric representations of solutions under different parameter conditions.
\end{abstract}

Keywords Coupled integrable system - Exact solution - Septic derivative nonlinear Schrödinger equation - Homoclinic orbits · Heteroclinic orbits . Periodic orbits

This research was partially supported by the National Natural Science Foundation of China (11471289, 11571318, 11162020).

\section{T. D. Leta $(\varangle) \cdot J$. Li}

Department of Mathematics, Zhejiang Normal University, Jinhua 321004, Zhejiang, People's Republic of China e-mail: leta@zjnu.cn

J. Li

School of Mathematical Sciences, Huaqiao University, Quanzhou 362021, Fujian, People's Republic of China e-mail: lijb@zjnu.cn

\section{Introduction}

Derivative nonlinear Schrödinger equations constitute a class of models which describe the evolution in physical media that has been drawn considerable attention both in a theoretical context and in many applied disciplines, notably in hydrodynamics, nonlinear optics and the study of Bose-Einstein condensates [1-4].

Moreover, nonlinear Schrödinger (NLS) equations incorporating cubic and quintic order nonlinearity are of fundamental interest in the physics of nonlinear optics $[4,5]$. It is natural to consider models in nonlinear dynamics combining these two features, and indeed such systems likewise have physical applications [6-8].

Recent interests on coupled nonlinear Schrödinger systems, on a class of propagating wave patterns for families of derivative nonlinear Schrödinger equations of seventh (septic), ninth (nonic) and thirteenth order, which incorporates de Broglie-Bohm quantum potentials and which admit integrable Ermakov-RayReid sub-systems have bought attention of researchers $[9,10]$.

In this paper, we shall consider the generalized class of higher-order derivative nonlinear Schrödinger equations of the type:

$$
\begin{aligned}
& i A_{t}+A_{x x}+f^{\prime}(\Phi) A+j^{\prime}(\Phi) i A_{x}+(g(\Phi))_{x} i A \\
& -\frac{s|A|_{x x}}{|A|} A=0
\end{aligned}
$$

where $(f, g$ and $j$ are arbitrary analytic functions which depend on the squared amplitude $\Phi=|A|^{2}=\phi^{2}$ 
$\left.+\psi^{2}\right)$, " " " stands for the derivative with respect to $\Phi$ and $s>1$ corresponds to the so-called resonant nonlinear Schrödinger equation because it admits both fission and fusion resonant solitonic phenomena [11,12].

A procedure recently employed by [13-16] (the application of a pair of invariants of motion) is also applied here. where $A^{2}$ be the squared amplitude, $x$ and $t$ are the normalized spatial coordinate and retarded time, respectively. To analyze the traveling wave solution with the form:

$$
\begin{aligned}
A(x, t) & =[(\phi(\xi)+i \psi(\xi))] \exp (i(v x-\lambda t)), \\
\xi & =x-c t
\end{aligned}
$$

where $c, v$, and $\lambda$; are related to the nonlinearity induced shifts in three quantities, namely, group delay, carrier frequency and propagation constant respectively. Substituting Eq. (2) into Eq. (1), separating the real and imaginary parts, one obtains the coupled nonlinear integrable system

$$
\begin{aligned}
\frac{\mathrm{d} \phi}{\mathrm{d} \xi}= & q_{1} \\
\frac{\mathrm{d} q_{1}}{\mathrm{~d} \xi}= & -\left(f^{\prime}+\lambda-v^{2}\right) \phi+\left(j^{\prime}+2 v-\mu\right) \dot{\psi} \\
& +g^{\prime}\left(\phi^{2}+\psi^{2}\right)^{\bullet} \psi \\
& +s\left[\frac{(\dot{\phi} \phi+\dot{\psi} \psi)^{\bullet}}{\phi^{2}+\psi^{2}}-\left(\frac{\dot{\phi} \phi+\dot{\psi} \psi}{\phi^{2}+\psi^{2}}\right)^{2}\right] \phi . \\
\frac{\mathrm{d} \psi}{\mathrm{d} \xi}= & q_{2}, \\
\frac{\mathrm{d} q_{2}}{\mathrm{~d} \xi}= & -\left(f^{\prime}+\lambda-v^{2}\right) \psi-\left(j^{\prime}+2 v-\mu\right) \dot{\phi} \\
& -g^{\prime}\left(\phi^{2}+\psi^{2}\right)^{\bullet} \phi \\
& +s\left[\frac{(\dot{\phi} \phi+\dot{\psi} \psi)^{\bullet}}{\phi^{2}+\psi^{2}}-\left(\frac{\dot{\phi} \phi+\dot{\psi} \psi}{\phi^{2}+\psi^{2}}\right)^{2}\right] \psi .
\end{aligned}
$$

where dot indicates a derivative with respect to $x-\mu t$.

Substituting

$f=\frac{a c}{\lambda} \Phi^{4}+\frac{\mu}{2} \Phi^{2}+\frac{v}{3} \Phi^{3}, j=2 a \Phi^{4}, \quad g=\frac{\delta}{2} \Phi^{2}$,

in system (3), that admits two independent integrals of motion and, accordingly, is integrable. Thus, the first integral becomes:

$$
\begin{aligned}
& \hat{H}\left(\phi, \psi, q_{1}, q_{2}\right)=\frac{1}{2}\left[q_{1}^{2}+q_{2}^{2}+\frac{a}{\lambda^{2}}(\mu-2 v) \Phi^{4}\right. \\
& \left.-\frac{v}{3 \lambda} \Phi^{3}+\left(\frac{\mu}{4 \lambda}+\frac{p \delta}{2 \lambda^{2}}\right) \Phi^{2}-\left(\lambda-v^{2}\right) \Phi-\frac{s}{4} \frac{\dot{\Phi}^{2}}{\Phi}\right]
\end{aligned}
$$

where the constant $\hat{H}=\hat{h}_{1}$ corresponds to the Hamiltonian invariant.

And the second integral

$$
\begin{aligned}
I\left(\phi, \psi, q_{1}, q_{2}\right)= & q_{1} \psi-q_{2} \phi-\frac{a}{\lambda^{2}} \Phi^{4} \\
& -\frac{\delta}{4 \lambda^{2}} \Phi^{2}+\frac{1}{2}(\mu-2 \nu) \Phi,
\end{aligned}
$$

where $I\left(\phi, \psi, q_{1}, q_{2}\right)=\hat{h}_{2}$ is constant of motion. The pair of integrals of motion (5) and (6) allow explicit solution of the nonlinear coupled system (3) for $\phi$ and $\psi$ in terms of quadrature.

It is easy to see that system (3) has at most 17 equilibrium points at $P_{0}(0,0,0,0), P_{a_{j}}\left( \pm \phi_{r_{j}}, 0,0,0\right)$, $P_{b_{j}}\left(0, \pm \psi_{r_{j}}, 0,0\right), j=1,2,3,4$, where $\phi_{r_{j}}=\psi_{r_{j}}=$ $\sqrt{X_{j}}$ are four positive real roots of the algebraic equation $\bar{\delta}_{1} \Phi^{4}-\bar{\delta}_{2} \Phi^{3}+\bar{\delta}_{3} \Phi^{2}-\bar{\delta}_{4} \Phi+\bar{\delta}_{0}=0$ Let $N_{\hat{h}_{1} \hat{h}_{2}}$ be an invariant manifold family of system (3) given by

$N_{\hat{h}_{1} \hat{h}_{2}}=\left\{\hat{H}=\hat{h}_{1}, I=\hat{h}_{2}, \hat{h}_{1}, \hat{h}_{2} \in \mathbf{R}\right\} \subset \mathbf{R}^{4}$.

Using the identity

$\left(\phi^{2}+\psi^{2}\right)\left(\dot{\phi}^{2}+\dot{\phi}^{2}\right)-(\phi \dot{\phi}+\psi \dot{\psi})^{2} \equiv(\phi \dot{\psi}-\psi \dot{\phi})^{2}$

combined with (5) and (6), for fixed $\left(\hat{h}_{1}, \hat{h}_{2}\right)$ gives rise

$$
\begin{aligned}
& \left(\frac{\mathrm{d} \Phi}{\mathrm{d} \xi}\right)^{2}=\frac{4}{1-s} \Phi\left[2 \hat{h}_{1}-\left(\lambda-v^{2}\right) \Phi\right. \\
& \left.+\left(\frac{p \delta-\mu}{2 \lambda}\right) \Phi^{2}-\frac{v}{3 \lambda} \Phi^{3}-\frac{a}{\lambda}(\mu-2 \nu) \Phi^{4}\right] \\
& -\frac{4}{1-s}\left[\hat{h}_{2}-\frac{1}{2}(\mu-2 v) \Phi+\frac{\delta}{4 \lambda} \Phi^{2}+\frac{a}{\lambda} \Phi^{4}\right]^{2}
\end{aligned}
$$

For Eq. (1), Rogers and Chow [13] introduced the variable $\Theta=\arctan \Delta$. So that, $\cos \Theta=\frac{\psi}{\sqrt{\Phi}}, \sin \Theta=\frac{\phi}{\sqrt{\Phi}}$, where $\Delta=\frac{\phi}{\psi}$. We see from (5) that, 


$$
\begin{aligned}
\Theta(\xi)= & \int_{0}^{\xi} \frac{4 \lambda \hat{h}_{2}-2(\mu-2 v) \lambda \Phi+\delta \Phi^{2}+4 a \Phi^{4}}{4 \lambda \Phi} \mathrm{d} \xi \\
= & -\frac{1}{2}(\mu-2 \nu) \xi+\hat{h}_{2} \int_{0}^{\xi} \frac{\mathrm{d} \xi}{\Phi}+\frac{\delta}{4 \lambda} \int_{0}^{\xi} \Phi \mathrm{d} \xi \\
& +\frac{a}{\lambda} \int_{0}^{\xi} \Phi^{3} \mathrm{~d} \xi
\end{aligned}
$$

where $\xi$ is a dummy variable of integration. Thus, if we know $\Phi(\xi)$ and $\Theta(\xi)$ from Eqs. (9) and (10), then we may solve Eq. (1) and system (3) to obtain the following solutions:

$A(x, t)=i \sqrt{\Phi} \exp [-i \Theta+i(v x-\lambda t)]$

and

$\phi(\xi)=\sqrt{\Phi} \sin \Theta$ and $\psi(\xi)=\sqrt{\Phi} \cos \Theta$

The corresponding class of exact solutions of Eq. (1), is then given by Eq. (11). Let $\frac{\mathrm{d} \Phi}{\mathrm{d} \xi}=\frac{1}{2}(1-s) y$, then we have a system

$$
\begin{aligned}
\frac{\mathrm{d} \Phi}{\mathrm{d} \xi} & =\frac{1}{2}(1-s) y, \\
\frac{\mathrm{d} y}{\mathrm{~d} \xi} & =b_{0}-b_{1} \Phi-b_{2} \Phi^{2}-b_{3} \Phi^{3}-b_{5} \Phi^{5}-b_{7} \Phi^{7} .
\end{aligned}
$$

where

$$
\begin{aligned}
& b_{0}=2 \hat{h}_{1}+\hat{h}_{2}(\mu-2 v) ; \\
& b_{1}=\frac{1}{\lambda}\left[(\mu-2 v)^{2} \lambda+2 \delta \hat{h}_{2}+4\left(\lambda-v^{2}\right) \lambda\right] ; \\
& b_{2}=\frac{1}{4 \lambda}[6 \mu-6 p \delta-3 \delta(\mu-2 v)] ; \\
& b_{3}=\frac{1}{6 \lambda^{2}}\left[3 \delta^{2}+48 a \hat{h}_{2} \lambda+8 v^{2} \lambda\right] ; \\
& b_{5}=\frac{4}{2 \lambda^{2}} a \delta ; \\
& b_{7}=\frac{8}{\lambda^{2}} a^{2} .
\end{aligned}
$$

Clearly, system (13) is a six-parameter system depending on the nine-parameter group $\left(\lambda, \mu, a, s, v, \delta, p, \hat{h}_{1}\right.$, $\left.\hat{h}_{2}\right)$. It is abound with dynamical system. For a given parameter group $(\nu, \mu) \neq(0,0)$, we next take $\left(\hat{h}_{1}, \hat{h}_{2}\right)$ such that

$b_{0} \equiv 0, \quad \hat{h}_{1}=\frac{(2 v-\mu)}{4} \times \hat{h}_{2}$.

Then, system (13) reduces to

$$
\begin{aligned}
\frac{\mathrm{d} \Phi}{\mathrm{d} \xi} & =\frac{1}{2}(1-s) y \\
\frac{\mathrm{d} y}{\mathrm{~d} \xi} & =-b_{1} \Phi-b_{2} \Phi^{2}-b_{3} \Phi^{3}-b_{5} \Phi^{5}-b_{7} \Phi^{7},
\end{aligned}
$$

with the first integral

$$
\begin{aligned}
\tilde{H}(\Phi, y)= & \frac{1}{4}(1-s) y^{2}+\left[\frac{1}{2} b_{1} \Phi^{2}+\frac{1}{3} b_{2} \Phi^{3}\right. \\
& \left.+\frac{1}{4} b_{3} \Phi^{4}+\frac{1}{6} b_{5} \Phi^{6}+\frac{1}{8} b_{7} \Phi^{8}\right] .
\end{aligned}
$$

Equation (15) is equivalent to the planar dynamical system

$$
\begin{aligned}
\frac{\mathrm{d} \Phi}{\mathrm{d} \xi}= & \frac{1}{2}(1-s) y, \\
\frac{\mathrm{d} y}{\mathrm{~d} \xi}= & S_{p}(\Phi) \\
\equiv & -b_{1} \Phi-b_{p} \Phi^{p}-b_{2 p-1} \Phi^{2 p-1} \\
& -b_{2 p+1} \Phi^{3 p-2},
\end{aligned}
$$

clearly, for $p=2,3$, we have

$$
\begin{aligned}
& S_{2}(\Phi)=-\Phi\left(b_{1}+b_{2} \Phi+b_{3} \Phi^{2}+b_{5} \Phi^{4}\right), \\
& S_{3}(\Phi)=-\Phi\left(b_{1}+b_{3} \Phi^{2}+b_{5} \Phi^{4}+b_{7} \Phi^{6}\right) .
\end{aligned}
$$

We notice that the exact traveling wave solution and bifurcation of the cubic-quintic was previously analyzed in [16-18]. Though, in this paper we give the dynamical behavior and the exact solution for the case of the septic order nonlinearities in the invariant manifold $N_{\hat{h}_{1} \hat{h}_{2}}$ by using Eq. (9). In order to consider the dynamics for system (17), when we apply a transformation $\Phi=\varphi^{\frac{1}{p^{-1}}}$, and substituting $\dot{\varphi}=\frac{1}{2}(1-s) y$ in system (17), then we obtain a new system:

$$
\begin{aligned}
& \frac{\mathrm{d} \varphi}{\mathrm{d} \xi}=\frac{1}{2}(1-s) y, \\
& \frac{\mathrm{d} y}{\mathrm{~d} \xi}=\frac{(p-2)(1-s)^{2} y^{2}-4(p-1)^{2}\left(b_{1} \varphi^{2}+b_{p} \varphi^{3}+b_{2 p-1} \varphi^{4}+b_{2 p+1} \varphi^{5}\right)}{4(p-1) \varphi} .
\end{aligned}
$$


Obviously, system (18) is a singular traveling wave system of the first class [19-21] with a singular straight lines $\varphi=0$. Thus, by the theory of singular systems, if an orbit of system (18) contacts the singular straight line at the origin, then along this orbit a phase point only takes finite "time interval" to arrive at the origin. The existence of the singular straight line leads to a dynamical behavior of solutions. Now, consider the associated regular system of (18), which has the same invariant curve solutions given as:

$$
\begin{aligned}
\frac{\mathrm{d} \varphi}{\mathrm{d} \zeta}= & 2(1-s)(p-1) \varphi y, \\
\frac{\mathrm{d} y}{\mathrm{~d} \zeta}= & (p-2)(1-s)^{2} y^{2}-4(p-1)^{2} \varphi^{2} \\
& \times\left(b_{1}+b_{p} \varphi+b_{2 p-1} \varphi^{2}+b_{2 p+1} \varphi^{3}\right) .
\end{aligned}
$$

where $\mathrm{d} \xi=-2(p-1) \varphi d \zeta$, for $p \neq 1$.

For $s=2$, system (18) has the first integral

$$
\begin{gathered}
H_{p}(\varphi, y)=\frac{3}{2}(p-1) \varphi^{-\frac{2(p-2)}{p-1}} y^{2}-2(p-1)^{2} \varphi^{\frac{2}{p-1}} \\
\left(\frac{2 b_{2 p+1}}{3 p-1} \varphi^{3}+\frac{b_{2 p-1}}{p} \varphi^{2}+\frac{2 b_{p}}{p+1} \varphi+b_{1}\right)=h .
\end{gathered}
$$

For the corresponding traveling wave Eq. (1) and systems (3), the following problems are considered here. What are the dynamical behavior of traveling wave solutions? How do the traveling wave solutions depend on the parameters of these systems? As far as we know, these problems have not been considered before for septic order nonlinearity.

We shall consider the existence and dynamical behavior of the bounded traveling wave solutions of Eq. (1) and system (3) in different regions of their parametric spaces, by using the methods of dynamical systems developed by $[22,23]$. Then, by calculating Eq. (8) and applying in Eqs. (9) and (10), we obtain the exact solutions of Eq. (1) and system (3).

This paper is organized as follows. In Sect. 2, we consider the bifurcations of phase portraits of system (18) for $p=2$. Corresponding to some phase portraits given in Sect. 2, in Sect. 3, we give some possible exact solutions of Eq. (1), under different parametric conditions and different $h$ values. In Sect. 4, we consider the dynamical behavior and bifurcation of phase portraits of system (18) for $p=3$. Corresponding to some phase portraits given in Sect. 4 , in Sect. 5, we give some possible exact solutions of Eq. (1), under different parametric conditions. Lastly, in Sect. 6, we state the main result.

\section{Bifurcations of phase portraits of system (18) when $p=2$}

First, we consider system (18) which has a Hamiltonian for $p=2$ given as

$$
\begin{aligned}
H_{2}(\varphi, y) & =\frac{3}{2} y^{2}-2 \varphi^{2}\left[b_{1}+\frac{2}{3} b_{2} \varphi+\frac{1}{2} b_{3} \varphi^{2}+\frac{1}{5} b_{5} \varphi^{3}\right] \\
& \equiv h .
\end{aligned}
$$

Obviously, system (18) has always has an equilibrium point $E_{0}(0,0)$. Write, $P_{3}(\varphi)=b_{1}+b_{2} \varphi+b_{3} \varphi^{2}+b_{5} \varphi^{3}$. Clearly, $P_{3}^{\prime}(\varphi)=b_{2}+2 b_{3} \varphi+3 b_{5} \varphi^{2}$. When $\varphi=r_{1,2}=$ $\frac{-b_{3} \pm \sqrt{\Delta}}{3 b_{5}}, \Delta=b_{3}^{2}-3 b_{2}$, we have $P_{3}^{\prime}\left(r_{1,2}\right)=0$. To investigate the equilibrium points of (18), we need to find all zeros of the function $P_{3}(\varphi)$. The cubic polynomial $P_{3}(\varphi)$ has three simple real zeros if and only if

$$
\begin{aligned}
Q\left(b_{1}, b_{2}, b_{3}\right) \equiv & \frac{1}{27} b_{2}^{3}-\frac{1}{108} b_{2}^{2} b_{3}^{2}-\frac{1}{6} b_{1} b_{2} b_{3} \\
& +\frac{1}{4} b_{1}^{2}+\frac{1}{27} b_{1} b_{3}^{3}<0 .
\end{aligned}
$$

If $Q\left(b_{1}, b_{2}, b_{3}\right)=0$, then $P_{3}(\varphi)$ has one simple zero $\varphi_{1}$ and one double real zero. When $Q\left(b_{1}, b_{2}, b_{3}\right)>0$, $P_{3}(\varphi)$ has only one real zero.

For a given fixed $b_{1}$ in the $\left(b_{2}, b_{3}\right)$-parametric plane, the function $Q\left(b_{2}, b_{3}\right)=0$ defines a quartic curves shown in Fig. 1a, b, which has three branches and partitions the $\left(b_{2}, b_{3}\right)$-parameter plane in to three regions. It is easy to prove that for $b_{1}>0$, the curves defined by $Q\left(b_{2}, b_{3}\right)=0$ has a cusp point at $\left(3 b_{1}^{\frac{2}{3}}, 3 b_{1}^{\frac{1}{3}}\right)$. While for $b_{1}<0$, the curves defined by $Q\left(b_{2}, b_{3}\right)=0$ has a cusp point at $\left(3 b_{1}^{\frac{2}{3}},-3 b_{1}^{\frac{1}{3}}\right)$. In the regions (II), (III) for $b_{1}>0$ and regions $\hat{I I}, I \hat{I} I$ for $b_{1}<0$, there exists three real zeros of $P_{3}(\varphi)$.

Let $\hat{M}\left(\varphi_{j}, 0\right)$ be the coefficient matrix of the linearized system of (18) at an equilibrium point $E_{j}$. We have $J(0,0)=\operatorname{det} \hat{M}(0,0)=0, J\left(\varphi_{j}, 0\right)=$ $\operatorname{det} \hat{M}\left(\varphi_{j}, 0\right)=-8 \varphi_{j}^{2}\left(4 \phi_{j}^{3}+3 b_{2} \phi_{j}^{2}+2 b_{1} \phi_{j}+b_{0}\right)$. We write that $h_{0}=H_{2}(0,0)=0, h_{j}=H_{2}\left(\varphi_{j}, 0\right)$. By using the above information to do qualitative analysis, we have the following bifurcations of the phase portraits of system (12) with different cases, when $b_{1}<0$, and $b_{1}>0$, respectively, as follows. 
Fig. 1 The partition of the $\left(b_{2}, b_{3}\right)$-parametric plane of system (18). a $b_{0}>0$, b $b_{0}<0$

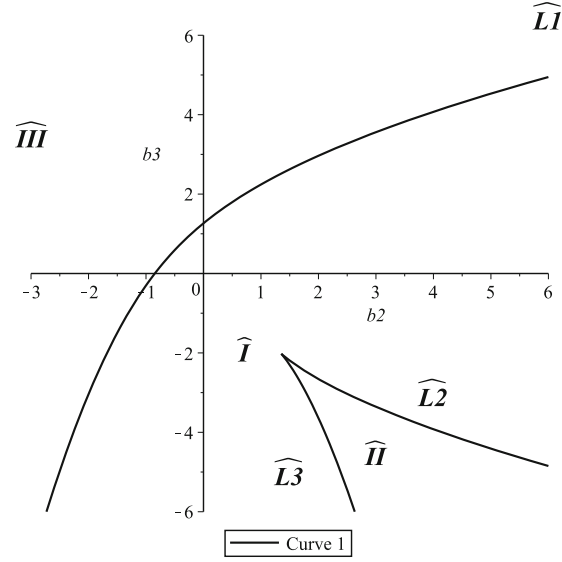

(a)

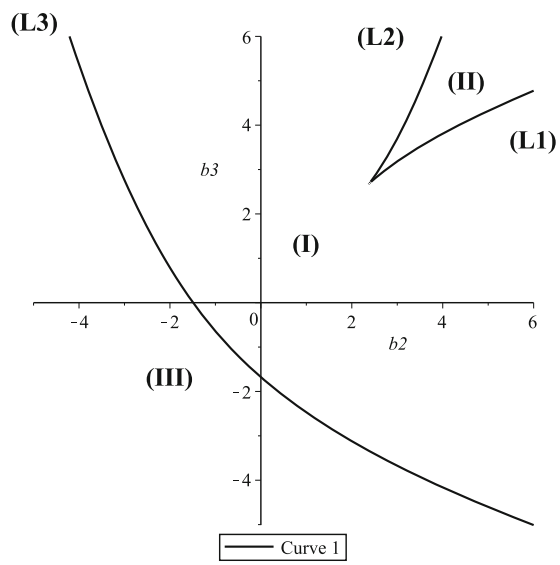

(b)

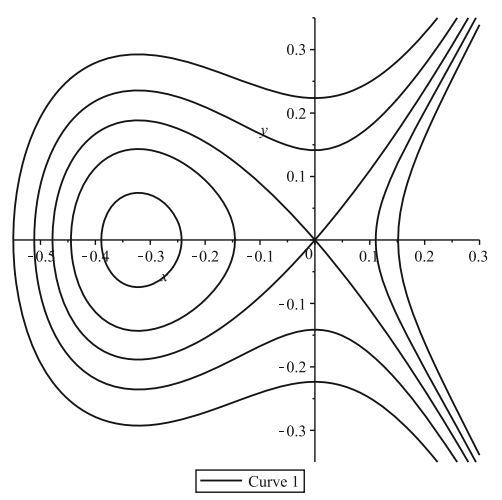

(a)

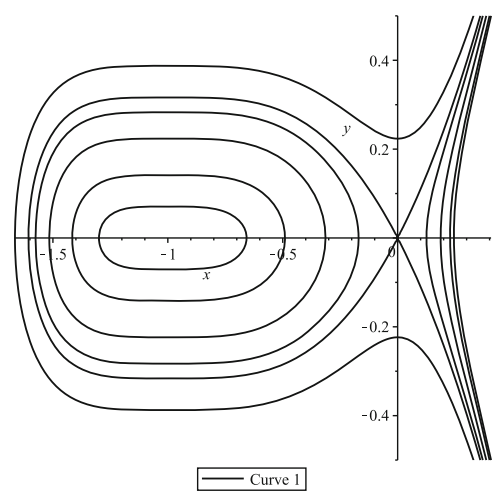

(b)

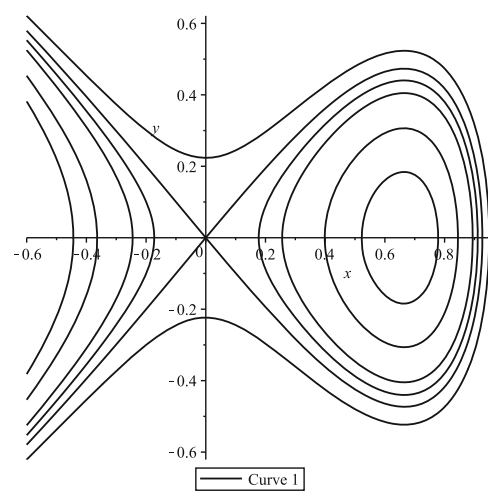

(c)

Fig. 2 Bifurcations of phase portraits of system (18), when $b_{1}>0$. a $r_{2}<0<r_{1}, h_{2}<0$, b $r_{2}=r_{1}<0, h_{1}=h_{2}<0$, c $0<r_{2}<r_{1}, b_{2}<h_{2}<0$

Case 1 Assume that $b_{1}>0, Q\left(b_{1}, b_{2}, b_{3}\right)<0$. When $b_{5}>0$, system (18) has two equilibrium points $E_{0}(0,0)$ and $E_{1}\left(\phi_{1}, 0\right)$. We have the phase portrait shown in Fig. 2a-c.

Case 2 Assume that $b_{1}>0, Q_{1}\left(b_{2}, b_{3}\right)<Q_{2}$ $\left(b_{2}, b_{3}\right)$. In this case, $P_{3}(\phi)$ has three real zeros $\phi_{j}, j=1,2,3$. System (18) has four equilibrium points $E_{0}(0,0)$ and $E_{j}\left(\phi_{j}, 0\right), j=1,2,3$. When $b_{5}>0$, the origin $E_{0}(0,0)$ and $E_{3}$ are saddle points; $E_{1}$ and $E_{2}$ are center points. The bifurcations of phase portraits of system (18) are shown in Fig. 3a-h.

Case 3 Assume that $b_{1}<0, Q_{1}\left(b_{2}, b_{3}\right)<0<$ $Q_{2}\left(b_{2}, b_{3}\right), 0<3 b_{2}<b_{3}^{2}$. System (18) has four equilibrium points $E_{0}(0,0)$ and $E_{j}\left(\phi_{j}, 0\right), j=1,2,3$. When $b_{5}>0$, the Equilibrium points $E_{1}$ and $E_{2}$ are saddle points; $E_{0}$ and $E_{3}$ are center points. The bifur- cations of phase portraits of system (18) are shown in Fig. 4a-h.

Case 4 Assume that $b_{1}<0,0<3 b_{2}<b_{3}^{2}, Q\left(b_{2}, b_{3}\right)>$ 0 . In this case, we have $\phi_{1}=\phi_{2}=r_{1} . P_{3}(\phi)$ has a double real zero $\phi_{12}=r_{1}$ and a simple real zero $\phi_{3}$. System (18) has three equilibrium points $E_{12}\left(r_{1}, 0\right), E_{0}(0,0)$ and $E_{3}\left(\phi_{3}, 0\right)$. When $b_{5}>0$, the origin $E_{0}(0,0)$ is center point, $E_{12}$ is a cusp and $E_{3}$ is a saddle point. The bifurcations of phase portraits of system (18) are shown in Fig. 5a-c.

\section{Explicit parametric representations of the solutions of system (18) when $p=2$}

We now consider the exact explicit parametric representations of the solutions of system (18) depending on 


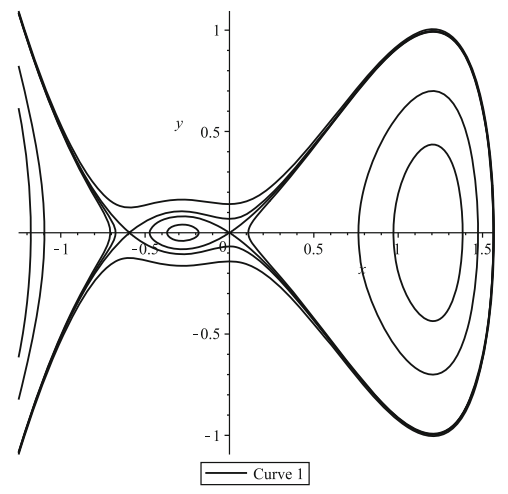

(a)

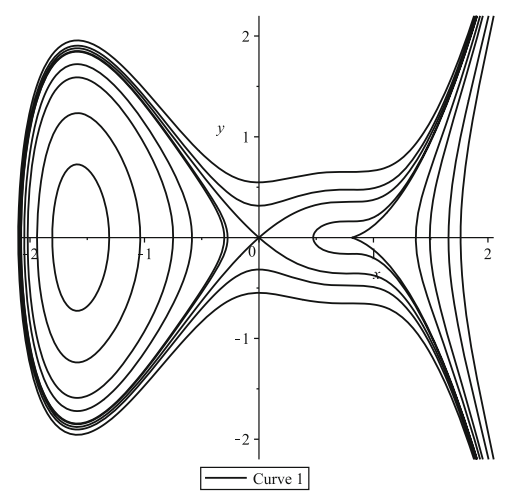

(d)

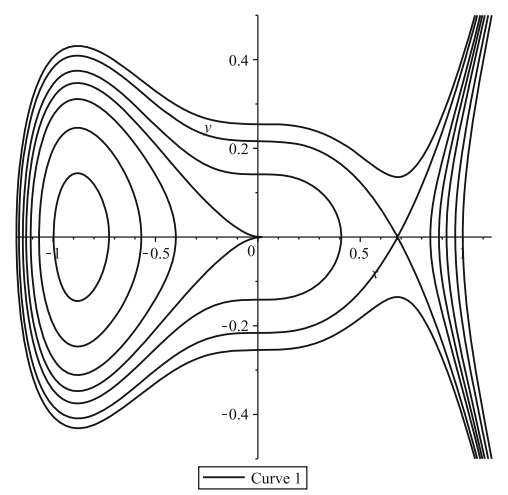

(g)

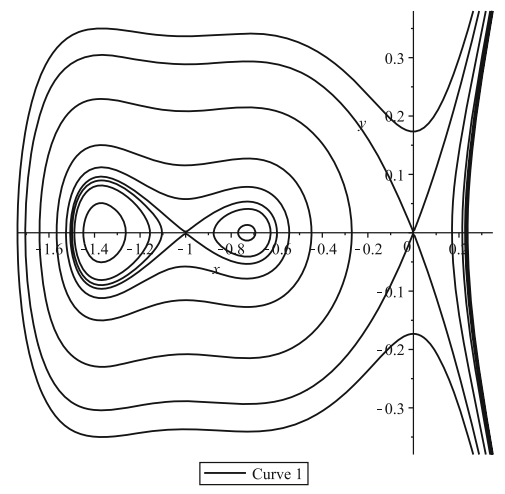

(b)

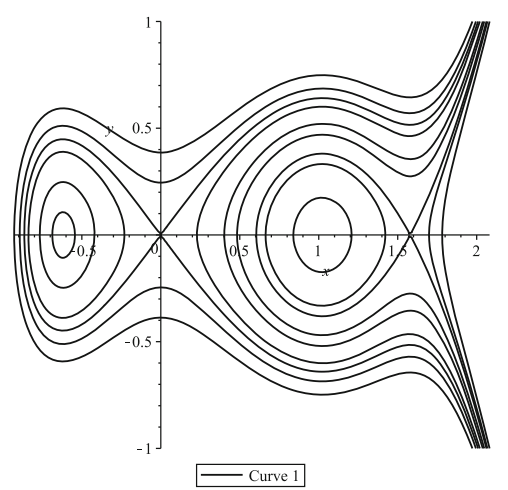

(e)

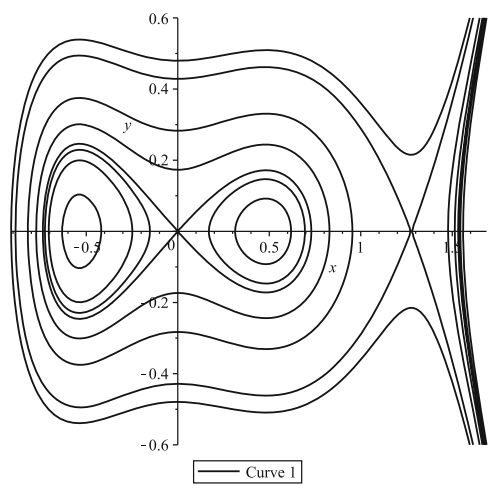

(h)

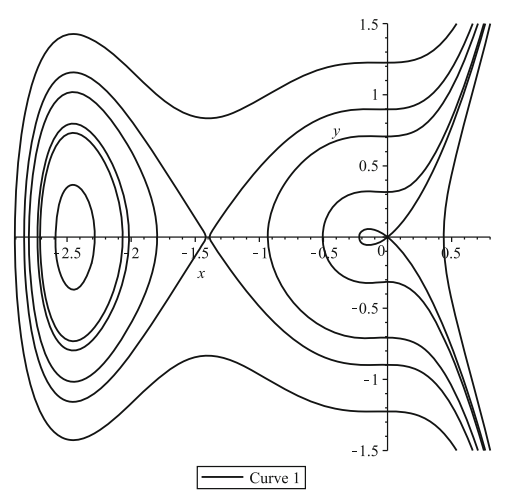

(c)

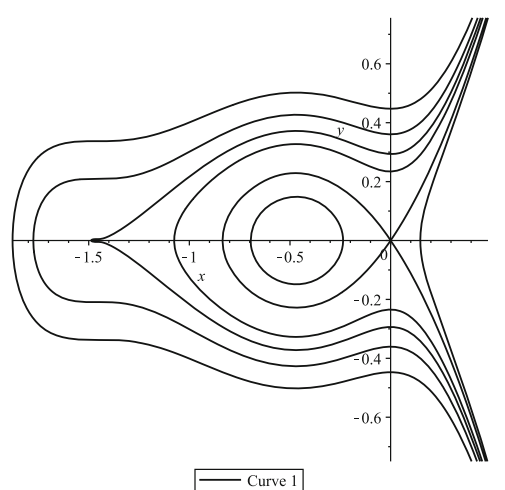

(f)

Fig. 3 Bifurcations of phase portraits of system (18), when $b_{1}>$ 0 . a $r_{2}<0<r_{1}, h_{1}<h_{2}<0$, b $r_{2}<r_{1}<0, h_{1}<h_{2}<0$, c $r_{2}<r_{1}<0,0<h_{2}<h_{1}, \mathbf{d} r_{2}<0<r_{1}, h_{2}=h_{3}$,

e $r_{1}<0<r_{2}, h_{2}<h_{1}<0, \mathbf{f} r_{1}<r_{2}<0, h_{1}=h 3, \mathbf{g}$ $r_{2}<0<r_{1}, h_{2}<0<h_{1}, \mathbf{h} r_{2}<0<r_{1}, h_{2}<0<h_{1}, \mathbf{i}$ $r_{2}=0<r_{1}, h_{1}<h_{2}=0$ 


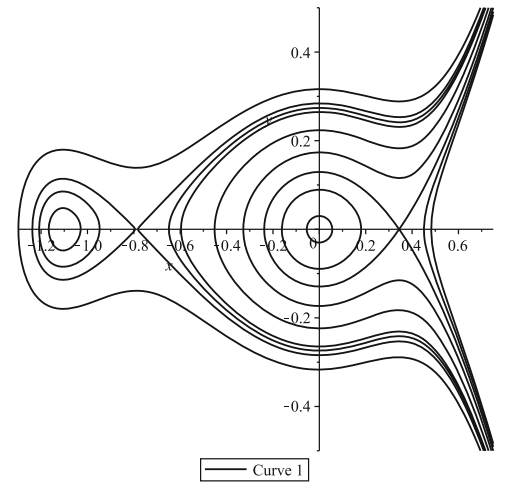

(a)

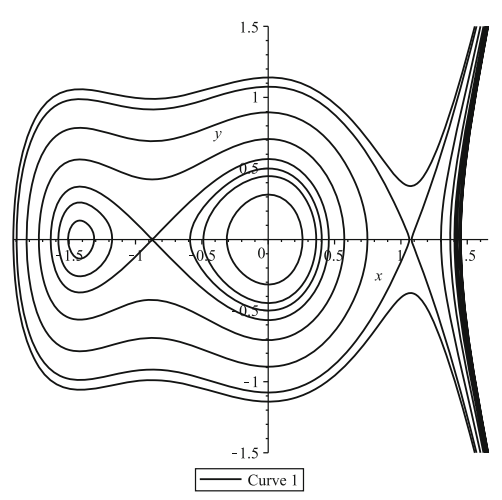

(d)

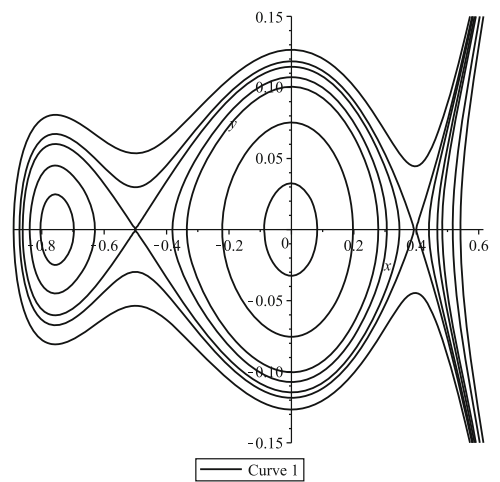

(b)

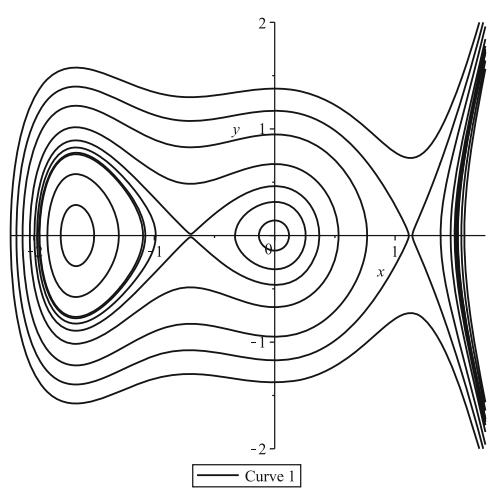

(e)

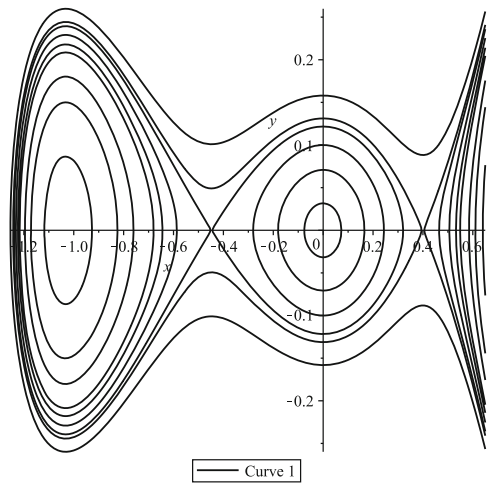

(c)

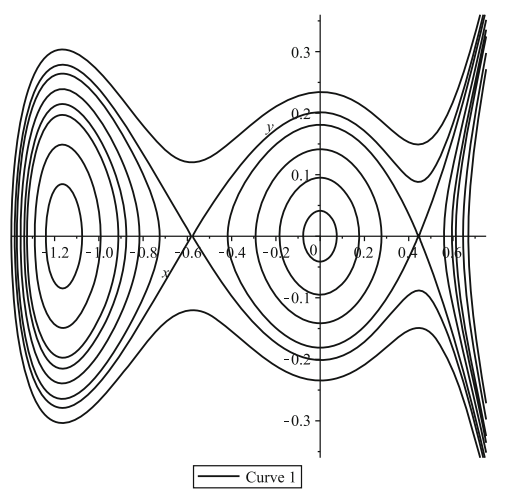

(f)

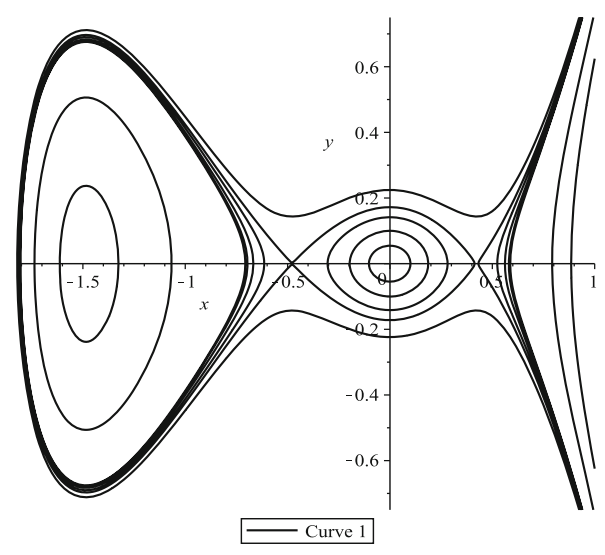

(g)

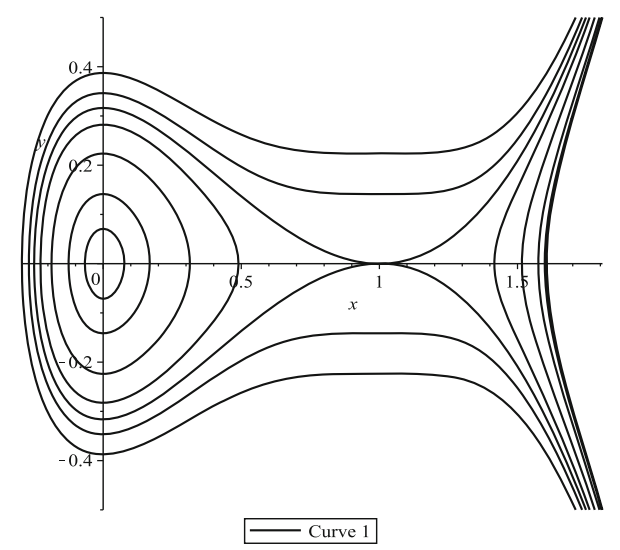

(h)

Fig. 4 Bifurcations of phase portraits of system (18), when $b_{1}<$ 0. a $r_{2}<0<r_{1}, h_{2}<0<h_{1}, \mathbf{b} r_{2}<b_{2}<r_{1}, 0<h_{1}<h_{2}$, c $r_{2}<0<r_{1}, h_{2}<0<h_{1}, \mathbf{d} r_{2}<0<r_{1}, 0<h_{1}<h_{2}$,

e $r_{2}<0<r_{1}, h_{2}<0<h_{1}, \mathbf{f} r_{2}<0<r_{1}, 0<h_{1}<h_{2}, \mathbf{g}$ $r_{1}<r_{2}<0,0<h_{1}<h_{2}, \mathbf{h} r_{2}=r_{1}>0, h_{1}=h_{2}>0$ 


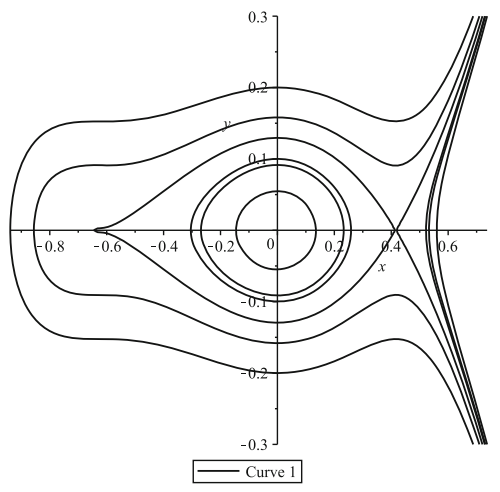

(a)

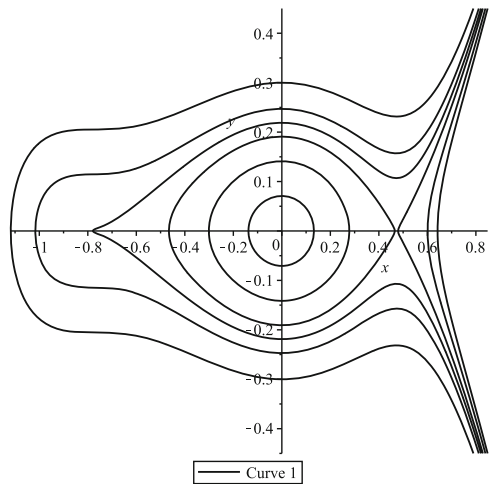

(b)

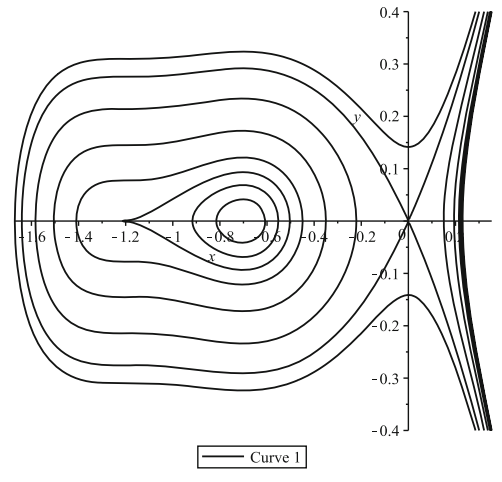

(c)

Fig. 5 Bifurcations of phase portraits of system (18) when $b_{1}<0$. a $h_{2}<r_{1}$, b $r_{1}<h_{2}, \mathbf{c} r_{2}<r_{1}<0, h_{2}=h_{3}<0$

$\sqrt{\Phi}$ and $\Theta$. We see from (21) and the first equation of system (18) that

$$
\begin{aligned}
\xi & =\int_{\Phi_{0}}^{\Phi} \frac{\mathrm{d} \Phi}{y(\Phi)} \\
& =\int_{\Phi_{0}}^{\Phi} \frac{\mathrm{d} \Phi}{\sqrt{\frac{2}{3} h+\frac{8}{15} b_{5} \Phi^{5}+\frac{2}{3} b_{3} \Phi^{4}+\frac{8}{9} b_{2} \Phi^{3}+\frac{4}{3} b_{1} \Phi^{2}}} .
\end{aligned}
$$

To find the exact solutions, we consider all bounded orbits of system (18) with $\Phi=\varphi>0$, for $p=2$. In this section, we discuss the exact solutions of Eq. (1) in the different regions of parameter plane $\left(b_{2}, b_{3}\right)$ for a given $b_{1}<0$ and $b_{1}>0$.

\subsection{Consider case 1 in Sect. 2 (see Fig. 2a, b)}

For $h=0$, the level curves defined by $H_{2}(\Phi, y)=$ $h, b_{1}>0$, there exists a homoclinic orbit of system (18) to the saddle point $E_{0}(0,0)$ enclosing the equilibrium point $E_{1}\left(\Phi_{1}, 0\right)$. In this case, (21) can be written as $y^{2}=\frac{4}{3} b_{1} \Phi^{2}+\frac{8}{9} b_{2} \Phi^{3}+\frac{2}{3} b_{3} \Phi^{4}+\frac{8}{15} b_{5} \Phi^{5}=$ $\left(\frac{8 b_{5}}{15}\right) \Phi^{2}\left(\Phi-\Phi_{m}\right)\left[\left(\Phi-\hat{b}_{1}\right)^{2}+\hat{a}_{1}^{2}\right]$. Hence, (21) implies we have the following parametric representation of $\Phi(\xi)$ :

$\Phi(\xi)=\left(\Phi_{m}+A_{1}\right)-\frac{2 A_{1}}{1+\operatorname{cn}\left(\omega_{0} \xi, k\right)}$.

Thus, we have from (10) that

$$
\begin{aligned}
\Theta(\xi)= & \left(\frac{1}{2}(2 v-\mu)+\frac{1}{2} \delta\left(\Phi_{m}+A_{1}\right)+\frac{a}{4}\left(\Phi_{m}+A_{1}\right)^{3}\right) \xi \\
& -\left(\delta+\frac{3 a}{2}\left(\Phi_{m}+A_{1}\right)^{2} A_{1}\right)
\end{aligned}
$$

$$
\begin{aligned}
& \times E\left(\arccos \left(\operatorname{cn}\left(\omega_{0} \xi, k\right)\right), k\right)-\frac{a}{4}\left(\Phi_{m}+A_{1}\right)^{3} \rho_{1} \\
& -3 a\left(\Phi_{m}+A_{1}\right) A_{1}^{2} \rho_{2}+\frac{\hat{h}_{2}}{\Phi_{m}+A_{1}} \Psi_{0} .
\end{aligned}
$$

Hence, we have the following solution Eq. (1):

$$
\begin{aligned}
A(x, t)= & i\left(\Phi_{m}+A_{1}-\frac{2 A_{1}}{1+\operatorname{cn}\left(\omega_{0} \xi, k\right)}\right)^{\frac{1}{2}} \\
& \times \exp [-i \Theta+i(v x-\lambda t)]
\end{aligned}
$$

where $\Theta(\xi)$ is given by (25), $A_{1}^{2}=\left(\hat{b}_{1}-\Phi_{m}\right)^{2}+$ $\hat{a}_{1}^{2}, k^{2}=\frac{A_{1}+\hat{b}_{1}-\Phi_{m}}{2 A_{1}}, \omega_{0}=\frac{\sqrt{8 b_{5}}}{\sqrt{15 A_{1}}}, \alpha_{r}^{2}=\frac{\Phi_{m}+A_{1}}{\Phi_{m}-A_{1}}$, $\Psi_{0}=\frac{\Phi_{m}-A_{1}}{2 \Phi_{m}}\left[\Pi\left(\arcsin \left(\operatorname{sn}\left(\omega_{0} \xi, k\right)\right), \frac{\alpha_{r}^{2}}{\alpha_{r}^{2}-1}, k\right)-\alpha_{r}^{2} \mathrm{sn}\right.$ $\left.\left(\omega_{0} \xi, k\right)\right], \rho_{1}=\frac{1}{5} k^{2} F\left(\arccos \left(\operatorname{cn}\left(\omega_{0} \xi, k\right)\right), k\right)-\frac{6}{5} k^{2}$ $E\left(\arccos \left(\operatorname{cn}\left(\omega_{0} \xi, k\right)\right), k\right)+\frac{2}{5}\left(4 k^{2}+1\right) \rho_{2}+$ $\frac{\operatorname{sn}\left(\omega_{0}, k\right) \operatorname{cn}\left(\omega_{0} \xi, k\right)}{5\left(1+\operatorname{cn}\left(\omega_{0} \xi, k\right)\right)^{3}}, \rho_{2}=\frac{1}{3}\left[\left(4 k^{2}+1\right) E\left(\arccos \left(\operatorname{cn}\left(\omega_{0} \xi\right.\right.\right.\right.$, $\left.k)), k)+\frac{\operatorname{sn}\left(\omega_{0} \xi, k\right) \operatorname{cn}\left(\omega_{0} \xi, k\right)}{\left(1+\operatorname{cn}\left(\omega_{0} \xi, k\right)\right)^{2}}\right]$.

3.2 Consider case 2 in Sect. 2 for $b_{1}>0$ (see Fig. 3a-h)

(1) The case of $r_{1}<0<r_{2} ; h_{3}<0$ (see Fig. 3a).

For $h_{2}=0$, the level curves defined by $H_{2}(\phi, y)$ $=0$, there exist two homoclinic orbits of system (18) with "eight shape" to the origin $E_{0}(0,0)$. In this case, (21) can be written as $y^{2}=\frac{8 b_{5}}{15} \Phi^{2}\left(\Phi-\Phi_{l}\right)(\Phi-$ $\left.\Phi_{m}\right)\left(\Phi_{M}-\Phi\right)$, where $\Phi_{l}<\Phi_{m}<\Phi_{1}<0<\Phi_{2}<$ $\Phi_{M}$.

Hence, for the right homoclinic orbit, we have the following parametric representation of $\Phi(\xi)$ :

$\Phi(\xi)=\Phi_{M}-\left(\Phi_{M}-\Phi_{m}\right) \mathrm{sn}^{2}\left(\omega_{1} \xi, k\right)$. 
Corresponding to the right homoclinic orbits we obtain from (10), that

$$
\begin{aligned}
\Theta(\xi)= & \left(\frac{1}{2}(2 v-\mu)+\frac{1}{2} \delta \Phi_{M}+\frac{a}{4} \Phi_{M}^{3}\right) \xi \\
& +\frac{\hat{h}_{2}}{\Phi_{M}} \Pi\left(\arcsin \left(\operatorname{sn}\left(\omega_{1} \xi, k\right)\right), \frac{\Phi_{M}-\Phi_{m}}{\Phi_{M}}, k\right) \\
& +\frac{1}{4}\left(3 a \Phi_{M}^{2}-2 \delta\right)\left(\Phi_{M}-\Phi_{m}\right) \beta_{1} \\
& -\frac{a}{4}\left[3 \Phi_{M} \beta_{2}+\left(\Phi_{M}-\Phi_{m}\right) \beta_{3}\right]\left(\Phi_{M}-\Phi_{m}\right)^{2}
\end{aligned}
$$

From Eqs. (27) and (28), we have the following solution of Eq. (1):

$$
\begin{aligned}
A(x, t)= & i\left(\Phi_{M}-\left(\Phi_{M}-\Phi_{m}\right) \operatorname{sn}^{2}\left(\omega_{1} \xi, k\right)\right)^{\frac{1}{2}} \\
& \times \exp [-i \Theta+i(\nu x-\lambda t)]
\end{aligned}
$$

where $\Theta(\xi)$ is given by (28), $\alpha_{1}^{2}=\frac{\Phi_{M}-\Phi_{m}}{\Phi_{M}}, k^{2}$ $=\frac{\Phi_{M}-\Phi_{m}}{\Phi_{M}-\Phi_{l}}, \omega_{1}=\sqrt{\frac{2 b_{5}\left(\Phi_{M}-\Phi_{l}\right)}{15}}$

$\beta_{1}=\frac{1}{k^{2}} E\left(\arcsin \left(\operatorname{sn}\left(\omega_{1} \xi, k\right)\right), k\right)$,

$\beta_{2}=\frac{1}{3 k^{2}} 2\left(1+k^{2}\right) \beta_{1}$ $+\frac{1}{3 k^{2}} \operatorname{sn}^{3}\left(\omega_{1} \xi, k\right) \operatorname{cn}\left(\omega_{1} \xi, k\right) \operatorname{dn}\left(\omega_{1} \xi, k\right)$,

$\beta_{3}=\frac{1}{5 k^{2}}\left[\operatorname{sn}^{3}\left(\omega_{1} \xi, k\right) \operatorname{cn}\left(\omega_{1} \xi, k\right) \operatorname{dn}\left(\omega_{1} \xi, k\right)\right.$

$$
\left.+4\left(1+k^{2}\right) \beta_{2}-3 \beta_{1}\right] \text {. }
$$

For the left homoclinic orbit, we have the following parametric representation of $\Phi(\xi)$ :

$\Phi(\xi)=\Phi_{l}-\frac{\Phi_{l}-\Phi_{m}}{\operatorname{dn}^{2}\left(\omega_{1} \xi, k\right)}$.

And, corresponding to the left homoclinic orbits we obtain from (10), that

$$
\begin{aligned}
& \Theta(\xi)=\left(\frac{1}{2}(2 v-\mu)+\frac{1}{2} \delta \Phi_{l}+\frac{a}{4} \Phi_{l}^{3}\right) \xi \\
& +\frac{\hat{h}_{2}}{\Phi_{l}}\left(\frac{\Phi_{l}}{\Phi_{m}}-1\right) \Pi\left(\arcsin \left(\operatorname{sn}\left(\omega_{1} \xi, k\right)\right),\right. \\
& \left.\quad \frac{\Phi_{l}}{\Phi_{m}} k^{2}, k\right)+\frac{1}{4}\left(2 \delta-3 a \Phi_{l}^{2}\right)\left(\Phi_{l}-\Phi_{m}\right) I_{1} \\
& +\left(\frac{3 a}{4} \Phi_{l} I_{2}-\frac{a}{4}\left(\Phi_{l}-\Phi_{m}\right) I_{3}\right)\left(\Phi_{l}-\Phi_{m}\right)^{2} .
\end{aligned}
$$

Hence, from Eqs. (30) and (31), we have the following solution of Eq. (1):

$$
\begin{aligned}
A(x, t)= & i\left(\Phi_{l}-\frac{\Phi_{l}-\Phi_{m}}{\operatorname{dn}^{2}\left(\omega_{1} \xi, k\right)}\right)^{\frac{1}{2}} \\
& \times \exp [-i \Theta+i(\nu x-\lambda t)]
\end{aligned}
$$

where $\Theta(\xi)$ is given by (31) and $I_{1}=\frac{1}{k^{\prime 2}} E(\arcsin$ $\left.\left(\operatorname{sn}\left(\omega_{1} \xi, k\right)\right), k\right)-\frac{k^{2}}{k^{\prime 2}} \operatorname{sn}\left(\omega_{1} \xi, k\right) \operatorname{cn}\left(\omega_{1} \xi, k\right), I_{2}=\frac{1}{k^{\prime 4}}$ $\left[2\left(2-k^{2}\right) E\left(\arcsin \left(\operatorname{sn}\left(\omega_{1} \xi, k\right)\right), k\right)-k^{2} \operatorname{sn}\left(\omega_{1} \xi, k\right) \mathrm{cn}\right.$ $\left.\left(\omega_{1} \xi, k\right)\left(k^{2} \mathrm{nd}^{2}\left(\omega_{1} \xi, k\right)+4-2 k^{2}\right)\right], I_{3}=\frac{1}{5 k^{\prime 2}}(5-$ $\left.4 k^{2}\right) I_{2}, k^{\prime}=\sqrt{1-k^{2}}$ and $\operatorname{sn}(\cdot, k), \mathrm{cn}(\cdot, k), \operatorname{dn}(\cdot, k)$, sd $(\cdot, k)$ are Jacobin elliptic functions and $\Pi(\cdot, \cdot, k)$, is the elliptic integrals of the third kind [24].

(2) The case of $r_{2}<r_{1}<0, h_{1}<h_{2}<0$ (see Fig. 3b).

Corresponding to the level curve defined by $H_{2}(\Phi, y)$ $=h, h \in\left(h_{1}, h_{2}\right)$, there exist two homoclinic orbit to the equilibrium $E_{2}\left(\Phi_{2}, 0\right)$ with "figure eight" of system (18). Now, (23) has the form

$$
\begin{aligned}
& \left(\sqrt{\frac{8 b_{5}}{15}}\right) \xi \\
& =\int_{\Phi}^{\Phi_{M}} \frac{\mathrm{d} \Phi}{\left(\Phi-\Phi_{2}\right) \sqrt{\left(\Phi-\Phi_{m}\right)\left(\Phi_{M}-\Phi\right)\left(\Phi_{L}-\Phi\right)}},
\end{aligned}
$$

and

$$
\begin{aligned}
& \left(\sqrt{\frac{8 b_{5}}{15}}\right) \xi \\
& =\int_{\Phi_{m}}^{\Phi} \frac{\mathrm{d} \Phi}{\left(\Phi_{2}-\Phi\right) \sqrt{\left(\Phi-\Phi_{m}\right)\left(\Phi_{M}-\Phi\right)\left(\Phi_{L}-\Phi\right)}},
\end{aligned}
$$

where $\Phi_{m}<\Phi_{1}<\Phi_{2}<\Phi_{3}<\Phi_{M}<0<\Phi_{L}$. Using the above integrals, corresponding to the right homoclinic orbit, we have the following parametric representation of $\Phi(\xi)$ :

$\Phi(\xi)=\Phi_{L}+\frac{\Phi_{M}-\Phi_{L}}{\mathrm{dn}^{2}\left(\omega_{2} \xi, k\right)}$.

Thus, corresponding to the right homoclinic orbits we obtain from (10), that

$$
\begin{aligned}
& \Theta(\xi)=\left(\frac{1}{2}(2 v-\mu)+\frac{1}{2} \delta \Phi_{L}+\frac{a}{4} \Phi_{L}^{3}\right) \xi \\
& +\frac{\hat{h}_{2}}{\Phi_{L}}\left(\frac{\Phi_{L}}{\Phi_{M}}-1\right) \Pi\left(\arcsin \left(\operatorname{sn}\left(\omega_{2} \xi, k\right)\right), \alpha_{2}^{2}, k\right) \\
& \quad-\frac{1}{4}\left(2 \delta-3 a \Phi_{L}^{2}\right)\left(\Phi_{M}-\Phi_{L}\right) I_{4} \\
& +\frac{3 a}{4} \Phi_{L}\left(\Phi_{M}-\Phi_{L}\right)^{2} I_{5}-\left(\Phi_{M}-\Phi_{L}\right)^{3} I_{6}
\end{aligned}
$$




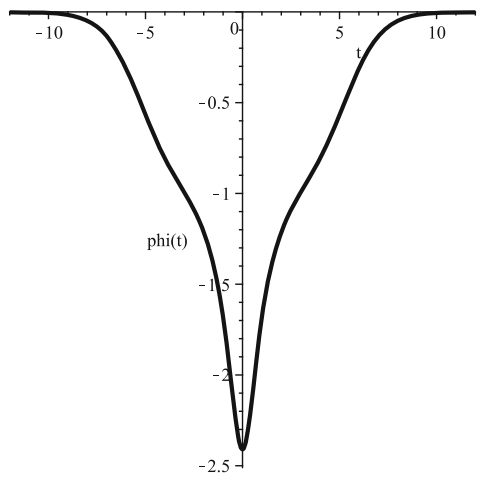

(a)

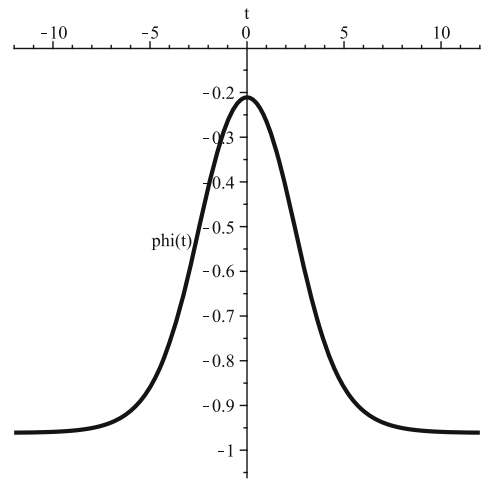

(b)

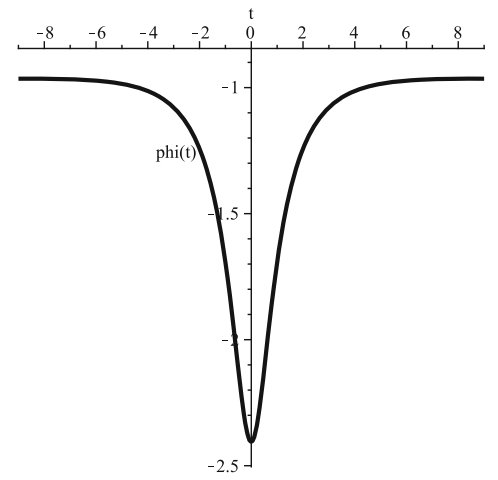

(c)

Fig. 6 Three profiles of the solitary waves of system (18). a $H_{2}(\Phi, y)=0, \mathbf{b} H_{2}(\Phi, y)=h_{2}$, right orbit, c $H_{2}(\Phi, y)=h_{2}$, left orbit

Hence, we have the following solution of Eq. (1):

$$
\begin{aligned}
A(x, t)= & i\left(\Phi_{L}+\frac{\Phi_{M}-\Phi_{L}}{\operatorname{dn}^{2}\left(\omega_{2} \xi, k\right)}\right)^{\frac{1}{2}} \\
& \times \exp [-i \Theta+i(\nu x-\lambda t)],
\end{aligned}
$$

where $\Theta(\xi)$ is given by (34) and $\alpha_{2}^{2}=\frac{\Phi_{M}-\Phi_{m}}{\Phi_{2}-\Phi_{m}}, I_{4}=$ $\frac{k^{2}}{k^{\prime 2}}\left[\beta_{4}-\operatorname{sn}\left(\omega_{2} \xi, k\right) \operatorname{cn}\left(\omega_{2} \xi, k\right)\right], I_{5}=\frac{k^{2}}{k^{\prime 4}} 2\left(2-k^{2}\right) \beta_{4}-$ $\frac{k^{2}}{k^{\prime 4}} \operatorname{sn}\left(\omega_{2} \xi, k\right) \operatorname{cn}\left(\omega_{2} \xi, k\right)\left(k^{2}\left(\mathrm{nd}^{2}\left(\omega_{2} \xi, k\right)-2\right)+4\right), I_{6}=$ $\frac{1}{5 k^{\prime 2}}\left(5-4 k^{2}\right) I_{5}$.

Corresponding to the left homoclinic orbit, we have the following parametric representation of $\Phi(\xi)$ :

$\Phi(\xi)=\Phi_{m}+\left(\Phi_{M}-\Phi_{m}\right) \mathrm{sn}^{2}\left(\omega_{2} \xi, k\right)$.

Thus we obtain from (10) that

$$
\begin{aligned}
& \Theta(\xi)=\left(\frac{1}{2}(2 v-\mu)+\frac{1}{2} \delta \Phi_{M}+\frac{a}{4} \Phi_{M}^{3}\right) \xi \\
& +\frac{\hat{h}_{2}}{\Phi_{m}} \Pi\left(\arcsin \left(\operatorname{sn}\left(\omega_{2} \xi, k\right)\right), \frac{\Phi_{m}-\Phi_{M}}{\Phi_{M}}, k\right) \\
& +\frac{1}{4}\left(2 \delta-3 a \Phi_{M}^{2}\right)\left(\Phi_{M}-\Phi_{m}\right) \beta_{4} \\
& \quad+\frac{a}{4}\left[3 \Phi_{M} \beta_{5}-\left(\Phi_{M}-\Phi_{m}\right) \beta_{6}\right]\left(\Phi_{M}-\Phi_{m}\right)^{2} .
\end{aligned}
$$

Thus, we have the following solution of Eq. (1):

$$
\begin{aligned}
A(x, t)= & i\left(\Phi_{m}+\left(\Phi_{M}-\Phi_{m}\right) \operatorname{sn}^{2}\left(\omega_{2} \xi, k\right)\right)^{\frac{1}{2}} \\
& \times \exp [-i \Theta+i(v x-\lambda t)],
\end{aligned}
$$

where $\Theta(\xi)$ is given by (37), and $k^{2}=\frac{\Phi_{M}-\Phi_{m}}{\Phi_{2}-\Phi_{m}}, \omega_{2}=$ $\sqrt{\frac{2 b_{5}\left(\Phi_{L}-\Phi_{m}\right)}{15}}, \beta_{4}=\frac{1}{k^{2}} E\left(\arcsin \left(\operatorname{sn}\left(\omega_{2} \xi, k\right)\right), k\right), \beta_{5}=$ $\frac{1}{3 k^{2}} 2\left(1+k^{2}\right) \beta_{4}+\frac{1}{3 k^{2}} \operatorname{sn}^{3}\left(\omega_{2} \xi, k\right) \operatorname{cn}\left(\omega_{2} \xi, k\right) \operatorname{dn}\left(\omega_{2} \xi, k\right)$, $\beta_{6}=\frac{1}{5 k^{2}} \operatorname{sn}^{3}\left(\omega_{2} \xi, k\right) \operatorname{cn}\left(\omega_{2} \xi, k\right) \operatorname{dn}\left(\omega_{2} \xi, k\right)+\frac{4}{5 k^{2}}(1+$ $\left.k^{2}\right) \beta_{5}-3 \beta_{4}$. Equations (33) and (36) give rise to the profiles of solitary waves shown in Fig. 6b, c.

(3) The case of $r_{2}<r_{1}<0, h_{1}=0$ (see Fig. 3c).

(i) Corresponding to the level curves defined by $H_{2}(\Phi, y)=0$, there exist a periodic orbits of system (18). (23) has the form

$\omega_{3} \xi=\int_{r_{2}}^{\Phi} \frac{\mathrm{d} \Phi}{\Phi \sqrt{\left(\Phi-r_{1}\right)\left(r_{2}-\Phi\right)\left(\Phi_{m}-\Phi\right)}}$,

where, $r_{2}<\Phi_{1}<r_{1}<\Phi_{m}<\Phi_{2}<0$. It gives rise to the parametric representations as follows:

$\Phi(\xi)=r_{1}+\left(r_{2}-r_{1}\right) \operatorname{sn}^{2}\left(\omega_{3} \xi, k\right)$.

From Eq. (10) we have

$$
\begin{aligned}
\Theta(\xi)= & \left(\frac{1}{2}(2 v-\mu)+\frac{1}{2} \delta r_{1}+\frac{a}{4} r_{1}^{3}\right) \xi \\
& +\frac{\hat{h}_{2}}{r_{1}} \Pi\left(\arcsin \left(\operatorname{sn}\left(\omega_{3} \xi, k\right)\right), \frac{r_{1}-r_{2}}{r_{1}}, k\right) \\
& -\frac{1}{4}\left(2 \delta+3 a r_{1}^{2}\right)\left(r_{1}-r_{2}\right) \beta_{7} \\
& -\frac{a}{4}\left[3 r_{2} \beta_{8}+\left(r_{2}-r_{1}\right) \beta_{9}\right]\left(r_{2}-r_{1}\right)^{2} .
\end{aligned}
$$

Hence, we have the following solution of Eq. (1):

$$
\begin{aligned}
A(x, t)= & i\left(r_{1}+\left(r_{2}-r_{1}\right) \operatorname{sn}^{2}\left(\omega_{3} \xi, k\right)\right)^{\frac{1}{2}} \\
& \times \exp [-i \Theta+i(v x-\lambda t)]
\end{aligned}
$$

where $\Theta(\xi)$ is given by $(40)$ and $k^{2}=\frac{r_{1}-r_{2}}{\Phi_{m}-r_{2}}, \omega_{3}=$ $\sqrt{\frac{2 b_{5}\left(\Phi_{m}-r_{2}\right)}{15}}$,

$\beta_{7}=\frac{1}{k^{2}} E\left(\arcsin \left(\operatorname{sn}\left(\omega_{3} \xi, k\right)\right), k\right)$, 


$$
\begin{aligned}
\beta_{8}= & \frac{1}{3 k^{2}} 2\left(1+k^{2}\right) \beta_{7} \\
& +\frac{1}{3 k^{2}} \operatorname{sn}^{3}\left(\omega_{3} \xi, k\right) \operatorname{cn}\left(\omega_{3} \xi, k\right) \operatorname{dn}\left(\omega_{3} \xi, k\right), \\
\beta_{9}= & \frac{1}{5 k^{2}} \operatorname{sn}^{3}\left(\omega_{3} \xi, k\right) \operatorname{cn}\left(\omega_{3} \xi, k\right) \operatorname{dn}\left(\omega_{3} \xi, k\right) \\
& +\frac{1}{5 k^{2}}\left[4\left(1+k^{2}\right) \beta_{8}-3 \beta_{7}\right] .
\end{aligned}
$$

(ii) Corresponding to the level curves defined by $H_{2}(\Phi, y)=0$, there exists a homoclinic orbit of system (18) to the saddle point $E_{0}(0,0)$ enclosing the equilibrium point $E_{2}\left(\Phi_{2}, 0\right)$. Equation (23) has the form

$$
\omega_{3} \xi=\int_{\Phi_{m}}^{\Phi} \frac{\mathrm{d} \Phi}{\Phi \sqrt{\left(\Phi-r_{1}\right)\left(\Phi-r_{2}\right)\left(\Phi-\Phi_{m}\right)}} .
$$

It gives rise to the parametric representations a homoclinic orbit of system (18) as follows:

$\Phi(\xi)=r_{1}+\frac{\Phi_{m}-r_{1}}{1-\operatorname{sn}^{2}\left(\omega_{3} \xi, k\right)}$.

Thus we obtain from (10) that

$$
\begin{aligned}
\Theta(\xi)= & \left(\frac{1}{2}(2 v-\mu)+\frac{1}{2} \delta r_{1}+\frac{a}{4} r_{1}^{3}\right) \xi \\
& +\left(3 r_{1} \Gamma_{1}+\frac{a}{4}\left(\Phi_{m}-r_{1}\right) \Gamma_{2}\right)\left(\Phi_{m}-r_{1}\right)^{2} \\
& +\frac{\hat{h}_{2}}{\Phi_{m}} \Psi_{1}+\frac{1}{4}\left(3 a r_{1}^{2}+2 \delta\right)\left(\Phi_{m}-r_{1}\right) \\
& \Pi\left(\arcsin \left(\operatorname{sn}\left(\omega_{3} \xi, k\right)\right), \frac{r_{1}}{\Phi_{m}}, k\right) .
\end{aligned}
$$

Hence, from Eqs. (42) and (43), we have the following solution of Eq. (1):

$$
\begin{aligned}
A(x, t)= & i\left(r_{1}+\frac{\Phi_{m}-r_{1}}{1-\operatorname{sn}^{2}\left(\omega_{3} \xi, k\right)}\right)^{\frac{1}{2}} \\
& \times \exp [-i \Theta+i(\nu x-\lambda t)],
\end{aligned}
$$

where $\Gamma_{1}=\frac{2\left(2 k^{2}-1\right)}{3 k^{\prime 4}} E\left(\arcsin \left(\operatorname{sn}\left(\omega_{3} \xi, k\right)\right), k\right)$ $+\frac{\left(2-4 k^{2}+k^{\prime 2} \mathrm{nc}^{2}\left(\omega_{3} \xi, k\right)\right)}{3 k^{\prime 4}} \mathrm{dn}\left(\omega_{3} \xi, k\right) \operatorname{tn}\left(\omega_{3} \xi, k\right), \quad \Gamma_{2}$ $=\frac{1}{5 k^{\prime 2}}\left[\left(3 k^{2}+4\left(1-2 k^{2}\right)\right) \Gamma_{1}+\operatorname{dn}\left(\omega_{3} \xi, k\right) \operatorname{tn}\left(\omega_{3}\right.\right.$ $\left.\xi, k) \mathrm{nc}^{4}\left(\omega_{3} \xi, k\right)\right]$ and $\Phi_{1}$ is a function of $\xi$. Note: $\Psi_{1}=\int_{\Phi_{m}}^{\Phi} \frac{1-\mathrm{sn}^{2}\left(\omega_{3} \xi, k\right)}{1-\alpha_{3}^{2} \mathrm{sn}^{2}\left(\omega_{3} \xi, k\right)} \mathrm{d} \xi$.

(4) The case of $r_{2}<0<r_{1}, h_{2}=h_{3}$ (see Fig. 3d).

Corresponding to the curves defined by $H_{2}(\Phi, y)=$ $h h \in\left(0, h_{1}\right)$, we have $y^{2}=\left(\frac{8 b_{5}}{15}\right)\left(\Phi-r_{2}\right)\left(r_{1}-\right.$ $\Phi)\left(\Phi_{2}-\Phi\right)^{3}$, where $r_{2}<\Phi_{1}<r_{1}<0<\Phi_{2}=\Phi_{3}$. Equation (18) has a periodic orbit enclosing $E_{1}\left(\Phi_{1}, 0\right)$, which gives rise to a parametric representation of Eq. (1). Now, (23) has the form

$\omega_{4} \xi=\int_{r_{2}}^{\Phi} \frac{\mathrm{d} \Phi}{\left(\Phi_{2}-\Phi\right) \sqrt{\left(\Phi-r_{2}\right)\left(r_{1}-\Phi\right)\left(\Phi_{2}-\Phi\right)}}$.

Therefore, we obtain the following parametric representation of a periodic orbits:

$\Phi(\xi)=r_{2}+\left(r_{1}-r_{2}\right) \operatorname{sn}^{2}\left(\omega_{4} \xi, k\right)$.

Thus we obtain from (10) that

$$
\begin{aligned}
\Theta(\xi)= & \left(\frac{1}{2}(2 v-\mu)+\frac{1}{2} \delta r_{2}+\frac{a}{4} r_{2}^{3}\right) \xi \\
& +\frac{\hat{h}_{2}}{r_{2}} \Pi\left(\arcsin \left(\operatorname{sn}\left(\omega_{4} \xi, k\right)\right),\left(\frac{r_{2}-r_{1}}{r_{2}}\right), k\right) \\
& +\frac{1}{4}\left(2 \delta+3 a r_{2}^{2}\right)\left(r_{1}-r_{2}\right) \beta_{10} \\
& +\frac{a}{4}\left[3 r_{2} \beta_{11}+\left(r_{1}-r_{2}\right) \beta_{12}\right]\left(r_{1}-r_{2}\right)^{2} .
\end{aligned}
$$

Hence, we have the following solution of Eq. (1):

$$
\begin{aligned}
A(x, t)= & i\left(r_{2}+\left(r_{1}-r_{2}\right) \operatorname{sn}^{2}\left(\omega_{4} \xi, k\right)\right)^{\frac{1}{2}} \\
& \times \exp [-i \Theta+i(\nu x-\lambda t),]
\end{aligned}
$$

where $\Theta(\xi)$ is given by (46), $k^{2}=\frac{r_{1}-r_{2}}{\Phi_{2}-r_{2}}, \beta_{10}=$ $\frac{1}{k^{2}} E\left(\arcsin \left(\operatorname{sn}\left(\omega_{4} \xi, k\right)\right), k\right)$,

$$
\begin{aligned}
\omega_{4}= & \frac{4 \sqrt{2 b_{4}}}{\sqrt{15\left(\Phi_{2}-r_{2}\right)}}, \\
\beta_{11}= & \frac{1}{3 k^{2}}\left[2\left(1+k^{2}\right) \beta_{10}\right. \\
& \left.+\operatorname{sn}^{3}\left(\omega_{4} \xi, k\right) \operatorname{cn}\left(\omega_{4} \xi, k\right) \operatorname{dn}\left(\omega_{4} \xi, k\right)\right], \\
\beta_{12}= & \frac{1}{5 k^{2}} \operatorname{sn}^{3}\left(\omega_{4} \xi, k\right) \operatorname{cn}\left(\omega_{4} \xi, k\right) \operatorname{dn}\left(\omega_{4} \xi, k\right) \\
& +\frac{1}{5 k^{2}}\left[4\left(1+k^{2}\right) \beta_{11}-3 \beta_{10}\right] .
\end{aligned}
$$

(5) The case of $r_{2}<0<r_{1}, h_{2}<0<h_{1}$ (see Fig. 3g).

For $h=0$ and $3 b_{2} b_{5}<b_{3}^{2}$ the level curves defined by $H_{2}(\Phi, y)=0$, we have from Eq. (21), $y^{2}=$ $\left(\frac{8 b_{5}}{15}\right)\left(\Phi-\Phi_{m}\right) \Phi^{3}\left(\Phi_{L}-\Phi\right)$, where $\Phi_{m}<\Phi_{1}<0<$ $\Phi_{L}$. Equation (18) has a degenerate homoclinic orbit at a cusp $E_{0}(0,0)$, enclosing $E_{1}\left(\Phi_{1}, 0\right)$. Now, (23) has the form

$\omega_{2} \xi=\int_{\Phi_{m}}^{\Phi} \frac{\mathrm{d} \Phi}{\Phi \sqrt{\left(\Phi-\Phi_{m}\right) \Phi\left(\Phi_{L}-\Phi\right)}}$. 
Therefore, we have the following parametric representation of $\Phi(\xi)$ :

$\Phi(\xi)=\Phi_{m}\left(1-\operatorname{sn}^{2}\left(\omega_{2} \xi, k\right)\right)$.

Thus we obtain from (10) that

$$
\begin{aligned}
\Theta(\xi)= & \left(\frac{1}{2}(2 v-\mu)+\frac{1}{2} \delta \Phi_{m}+\frac{a}{4} \Phi_{m}^{3}\right) \xi \\
& -\frac{1}{4}\left(2 \delta+3 a \Phi_{m}^{3}\right) I_{7}+\frac{a}{4} \Phi_{m}^{3}\left(3 I_{8}-I_{9}\right) \\
& +\frac{\hat{h}_{2}}{\Phi_{m}}\left(E\left(\arcsin \left(\operatorname{sn}\left(\omega_{2} \xi, k\right)\right), k\right)\right. \\
& \left.-\operatorname{dn}\left(\omega_{2} \xi, k\right) \operatorname{tn}\left(\omega_{2} \xi, k\right)\right)
\end{aligned}
$$

Therefore, we have the following solution of Eq. (1):

$$
\begin{aligned}
A(x, t)= & i \sqrt{\Phi_{m}}\left(\left(1-\operatorname{sn}^{2}\left(\omega_{2} \xi, k\right)\right)\right)^{\frac{1}{2}} \\
& \times \exp [-i \Theta+i(\nu x-\lambda t),]
\end{aligned}
$$

where $\Theta(\xi)$ is given by (49) and $k^{2}=\frac{\Phi_{m}}{\Phi_{L}-\Phi_{m}}, I_{7}=$ $\frac{1}{k^{2}} E\left(\arcsin \left(\operatorname{sn}\left(\omega_{2} \xi, k\right)\right), k\right), I_{8}=\frac{1}{3 k^{2}} 2\left(1+k^{2}\right) I_{7}+k^{2}$ $\operatorname{sn}^{3}\left(\omega_{2} \xi, k\right) \operatorname{cn}\left(\omega_{2} \xi, k\right) \operatorname{dn}\left(\omega_{2} \xi, k\right), I_{9}=\frac{1}{5 k^{2}} \operatorname{sn}^{3}\left(\omega_{2} \xi\right.$, $k) \operatorname{cn}\left(\omega_{2} \xi, k\right) \operatorname{dn}\left(\omega_{2} \xi, k\right)+\frac{1}{5 k^{2}}\left[4\left(1+k^{2}\right) I_{8}-3 I_{7}\right]$.

(6) The case of $r_{2}<0<r_{1}, h_{2}<0<h_{1}$ (see Fig. 3h).

Corresponding to the level curve defined by $H_{2}(\Phi, y)$ $=0$, there exist two homoclinic orbit to the origin $E_{0}(0,0)$ with "figure eight" of (18). Now, (23) has the form

$\omega_{2} \xi=\int_{\Phi}^{\Phi_{M}} \frac{\mathrm{d} \Phi}{\Phi \sqrt{\left(\Phi-\Phi_{m}\right)\left(\Phi_{M}-\Phi\right)\left(\Phi_{L}-\Phi\right)}}$

and

$\omega_{2} \xi=\int_{\Phi_{m}}^{\Phi} \frac{\mathrm{d} \Phi}{\Phi \sqrt{\left(\Phi-\Phi_{m}\right)\left(\Phi_{M}-\Phi\right)\left(\Phi_{L}-\Phi\right)}}$,

where $\Phi_{m}<\Phi_{1}<0<\Phi_{2}<\Phi_{M}<\Phi_{L}$. Using the above integrals, corresponding to the right and left homoclinic orbit we have the same parametric representation and solution of Eq. (1) as (35) and (38), respectively.

(7) The case of $r_{2}=0<r_{1}, h_{1}<h_{2}=0$ (see Fig. 3i).

In this case, corresponding to the level curve defined by $H_{2}(\Phi, y)=0$, there exists a homoclinic orbit to the origin $E_{0}(0,0)$, enclosing the equilibrium point $E_{1}\left(\Phi_{1}, 0\right)$ and there are two heteroclinic orbits connecting the equilibrium point $E_{3}\left(\Phi_{3}, 0\right)$ and $E_{0}(0,0)$, enclosing the equilibrium point $E_{2}\left(\Phi_{2}, 0\right)$. Now (23) can be written as

$\left(\sqrt{\frac{8 b_{5}}{15}}\right) \xi=\int_{\Phi_{m}}^{\Phi} \frac{\mathrm{d} \Phi}{\left(\Phi_{3}-\Phi\right) \Phi \sqrt{\Phi-\Phi_{m}}}$.

(i) Corresponding to the left homoclinic orbit, we have the following parametric representation of $\Phi(\xi)$ :

$\Phi(\hat{\xi})=\Phi_{m} \operatorname{sech}^{2}(\hat{\xi}), \quad \hat{\xi} \in(0, \infty)$,

we obtain from Eq. (10) that

$$
\begin{aligned}
\Theta(\hat{t})= & \frac{1}{2}(2 v-\mu) \hat{t}+\frac{\delta}{2} \Phi_{m} \tanh (\hat{\xi}) \\
& +\frac{\hat{h}_{2}}{2 \Phi_{m}} \sinh (\hat{\xi}) \cosh (\hat{\xi})+\frac{a}{4} \Phi_{m}^{3} \\
& \times\left[\frac{\sinh (\hat{\xi})}{\cosh (\hat{\xi})}+\frac{4 \sinh (\hat{\xi})}{5 \cosh ^{3}(\hat{\xi})}+\frac{4 \sinh (\hat{\xi})}{15 \cosh ^{2}(\hat{\xi})}\right. \\
& \left.+\frac{1}{2} \ln \left(\frac{1+\sinh (\hat{\xi})}{1-\sinh (\hat{\xi})}\right)\right] .
\end{aligned}
$$

where, $\hat{\xi}=\tanh ^{-1} \sqrt{\frac{\Phi(\hat{\xi})-\Phi_{m}}{\Phi_{m}}}$. Hence, we have the following solution of Eq. (1):

$$
\begin{aligned}
A(x, t)= & i \sqrt{\Phi_{m}} \operatorname{sech}(\hat{t}) \\
& \times \exp [-i \Theta+i(v x-\lambda t),]
\end{aligned}
$$

where $\Theta(\hat{t})$ is given by (52).

(ii) Corresponding to the heteroclinic orbit, we have the following parametric representation of $\Phi(\xi)$ :

$\Phi(\hat{\xi})=\Phi_{m}+\left(\Phi_{m}-\Phi_{3}\right) \tanh ^{2}(\hat{t})$,

we have from Eq. (10) that

$$
\begin{aligned}
& \Theta(\hat{\xi})=\left(\frac{1}{2}(2 v-\mu)+\frac{1}{2} \delta \Phi_{m}+\frac{a}{4} \Phi_{m}^{3}\right) \hat{\xi} \\
& +\frac{\hat{h}_{2}}{\Phi_{3}} \sqrt{\frac{\Phi_{m}-\Phi_{3}}{\Phi_{m}}} \tanh ^{-1}\left(\sqrt{\frac{\Phi_{m}-\Phi_{3}}{\Phi_{m}}} \tanh (\hat{\xi})\right) \\
& +\frac{1}{4}\left[3 a \Phi_{m}^{2}-2 \delta\right]\left(\Phi_{m}-\Phi_{3}\right) \tanh (\hat{\xi}) \\
& \quad-\frac{3 a}{4}\left(\tanh ^{3}(\hat{\xi})-4 \tanh (\hat{\xi})\right) \Phi_{m}\left(\Phi_{m}-\Phi_{3}\right)^{2} \\
& -\frac{a}{4}\left(\frac{1}{5} \tanh ^{5}(\hat{\xi})-\frac{1}{4} \tanh ^{3}(\hat{\xi})-\tanh (\hat{\xi})\right) \\
& \left(\Phi_{3}-\Phi_{m}\right)^{3}
\end{aligned}
$$

where $\hat{\xi}=\tanh ^{-1} \sqrt{\frac{\Phi(\hat{\xi})-\Phi_{m}}{\Phi_{m}-\Phi_{3}}}$. Thus, we have the following solution of Eq. (1): 
Fig. 7 The profiles of kink and anti-kink waves of system (18). a Kink wave, b anti-kink wave

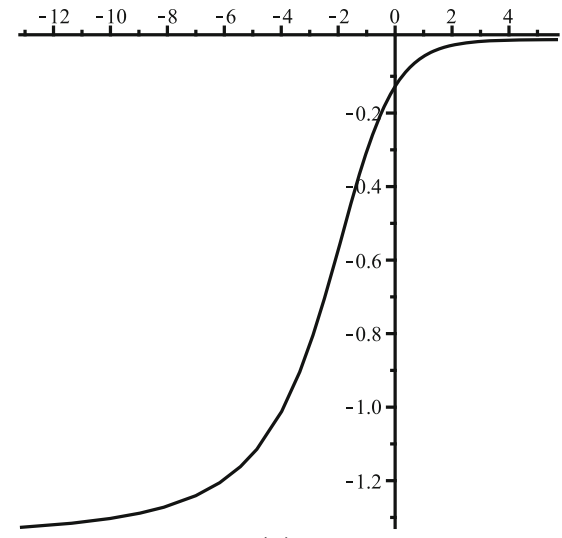

(a)

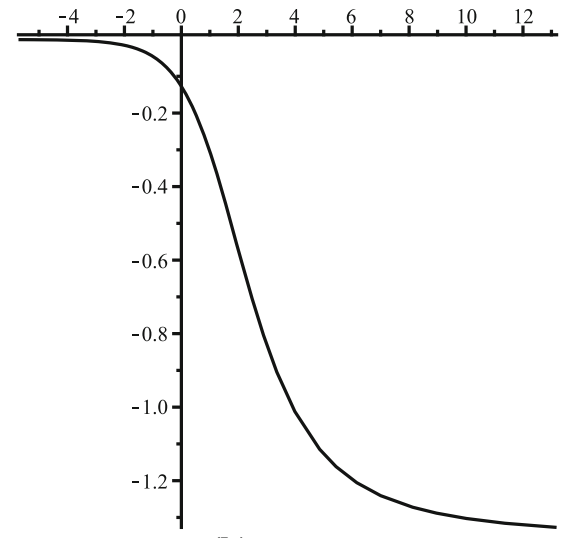

(b)

$$
\begin{aligned}
A(x, t)= & i\left(\Phi_{m}+\left(\Phi_{m}-\Phi_{3}\right) \tanh ^{2}(\hat{\xi})\right)^{\frac{1}{2}} \\
& \exp [-i \Theta+i(v x-\lambda t),]
\end{aligned}
$$

where $\Theta(\hat{\xi})$ is given by $(55)$.

Equation (54) give rise to the profiles of kink and anti-kink waves shown in Fig. 7a, b.

\subsection{Consider case 3 in section 2 , for $b_{1}<0$ (see}

Fig. $4 \mathrm{a}-\mathrm{h})$.

In this case, the phase portraits are determined by making a shift transformation, such that a saddle point becomes the origin, we can obtain the parametric representations of some solutions of heteroclinic and homoclinic orbits.

(1) The case of $r_{1}<r_{2}<0,0<h_{1}<h_{2}$ (see Fig. 4g).

For $h \in\left(h_{2},+\infty\right)$, the level curve defined by $H_{2}(\Phi, y)=h$, there are two heteroclinic orbits connecting the equilibrium point $E_{2}\left(\Phi_{2}, 0\right)$ and $E_{3}\left(\Phi_{3}, 0\right)$, enclosing the equilibrium point $E_{0}(0,0)$ and there exists a homoclinic orbit to the equilibrium point $E_{2}\left(\Phi_{2}, 0\right)$, enclosing the equilibrium point $E_{1}\left(\Phi_{1}, 0\right)$. Now (23) can be written as

$\left(\sqrt{\frac{8 b_{5}}{15}}\right) \xi=\int_{\Phi_{m}}^{\Phi} \frac{\mathrm{d} \Phi}{\left(\Phi_{3}-\Phi\right)\left(\Phi-\Phi_{2}\right) \sqrt{\Phi-\Phi_{m}}}$,

where, $\Phi_{m}<\Phi_{1}<\Phi_{2}<0<\Phi_{3}$.

(i) For the heteroclinic orbit, we have the following parametric representation of $\Phi(\xi)$ :

$$
\Phi(\hat{\xi})=\Phi_{m}+\left(\Phi_{2}-\Phi_{m}\right) \tanh ^{2}(\hat{\xi})
$$

we have from Eq. (10) that

$$
\begin{aligned}
\Theta(\hat{t})= & \left(\frac{1}{2}(2 v-\mu)+\frac{1}{2} \delta \Phi_{m}+\frac{a}{4} \Phi_{m}^{3}\right) \hat{\xi} \\
& +\frac{\hat{h}_{2}}{\Phi_{2}-2 \Phi_{m}} \sqrt{\frac{\Phi_{2}-\Phi_{m}}{\Phi_{m}}} \\
& \tanh ^{-1}\left(\sqrt{\frac{\Phi_{2}-\Phi_{m}}{\Phi_{m}}} \tanh (\hat{\xi})\right) \\
& +\frac{1}{4}\left[3 a \Phi_{m}^{2}-2 \delta\right]\left(\Phi_{m}-\Phi_{2}\right) \tanh (\hat{\xi}) \\
& -\frac{3 a}{4} \Phi_{m}\left(\frac{1}{4} \tanh ^{3}(\hat{\xi})-\tanh (\hat{\xi})\right)\left(\Phi_{m}-\Phi_{2}\right)^{2} \\
& +\frac{a}{4}\left(\frac{1}{5} \tanh ^{5}(\hat{\xi})-\frac{1}{4} \tanh ^{3}(\hat{\xi})-\tanh (\hat{\xi})\right) \\
& \left(\Phi_{m}-\Phi_{2}\right)^{3} .
\end{aligned}
$$

Hence, we have the following solution of Eq. (1):

$$
\begin{aligned}
A(x, t)= & i\left(\Phi_{m}+\left(\Phi_{2}-\Phi_{m}\right) \tanh ^{2}(\hat{\xi})\right)^{\frac{1}{2}} \\
& \exp [-i \Theta+i(\nu x-\lambda t)]
\end{aligned}
$$

where $\Theta(\hat{\xi})$ is given by (58) and $\hat{\xi}=\tanh ^{-1}$ $\sqrt{\frac{\Phi(\hat{\xi})-\Phi_{m}}{\Phi_{2}-\Phi_{m}}}$

(ii) Corresponding to the homoclinic orbit of system (18) to the equilibrium point $E_{2}\left(\Phi_{2}, 0\right)$ enclosing the equilibrium point $E_{1}\left(\phi_{1}, 0\right)$, we obtain a parametric representation of $\Phi(\xi)$ :

$\Phi(\hat{\xi})=\Phi_{m}+\left(\Phi_{m}-\Phi_{2}\right) \tanh ^{2}(\hat{\xi})$,

we have from Eq. (10) that

$$
\begin{aligned}
& \Theta(\hat{\xi})=\left(\frac{1}{2}(2 v-\mu)+\frac{1}{2} \delta \Phi_{m}+\frac{a}{4} \Phi_{m}^{3}\right) \hat{\xi} \\
& +\frac{\hat{h}_{2}}{\Phi_{2}} \sqrt{\frac{\Phi_{m}-\Phi_{2}}{\Phi_{m}}} \tanh ^{-1}\left(\sqrt{\frac{\Phi_{m}-\Phi_{2}}{\Phi_{m}}} \tanh (\hat{\xi})\right)
\end{aligned}
$$




$$
\begin{aligned}
& +\frac{1}{4}\left[3 a \Phi_{m}^{2}-2 \delta\right]\left(\Phi_{2}-\Phi_{m}\right) \tanh (\hat{\xi}) \\
& -\frac{3 a}{4} \Phi_{m}\left(\frac{1}{4} \tanh ^{3}(\hat{\xi})-\tanh (\hat{\xi})\right)\left(\Phi_{2}-\Phi_{m}\right)^{2} \\
& -\frac{a}{4}\left(\frac{1}{5} \tanh ^{5}(\hat{\xi})-\frac{1}{4} \tanh ^{3}(\hat{\xi})\right. \\
& -\tanh (\hat{\xi}))\left(\Phi_{2}-\Phi_{m}\right)^{3}
\end{aligned}
$$

Therefore, we have the following solution of Eq. (1):

$$
\begin{aligned}
A(x, t)= & i\left(\Phi_{m}+\left(\Phi_{m}-\Phi_{2}\right) \tanh ^{2}(\hat{\xi})\right)^{\frac{1}{2}} \\
& \exp [-i \Theta+i(\nu x-\lambda t)],
\end{aligned}
$$

where $\Theta(\hat{\xi})$ is given by (61) and $\hat{\xi}=\tanh ^{-1}$ $\sqrt{\frac{\Phi(\hat{\xi})-\Phi_{m}}{\Phi_{2}-\Phi_{m}}}$

(2) The case of $r_{2}=r_{1}>0, h_{1}=h_{2}>0$ (see Fig. 4h).

For $h=0.1$, the level curve defined by $H_{2}(\Phi, y)=$ $h$, there exists a degenerated homoclinic orbit to the multiple equilibrium point $\left(\Phi_{1}, 0\right),(23)$ becomes that $\left(\sqrt{\frac{8 b_{5}}{15}}\right) \xi=\int_{\Phi_{m}}^{\Phi} \frac{\mathrm{d} \Phi}{\left(\Phi_{1}-\Phi\right)^{2} \sqrt{\Phi-\Phi_{m}}}$,

where $\Phi_{m}<0<\Phi_{1}$. Thus, we have parametric representation:

$\Phi(\hat{\xi})=\Phi_{m}+\left(\Phi_{1}-\Phi_{m}\right) \tanh ^{2}(\hat{\xi})$,

we obtain from Eq. (10) that

$$
\begin{aligned}
& \Theta(\hat{\xi})=\left(\frac{1}{2}(2 \nu-\mu)+\frac{1}{2} \delta \Phi_{m}+\frac{a}{4} \Phi_{m}^{3}\right) \hat{\xi} \\
& +\frac{\hat{h}_{2}}{\Phi_{1}-2 \Phi_{m}} \sqrt{\frac{\Phi_{1}-\Phi_{m}}{\Phi_{m}}} \tanh ^{-1} \\
& \left(\sqrt{\left.\frac{\Phi_{1}-\Phi_{m}}{\Phi_{m}} \tanh (\hat{\xi})\right)}\right. \\
& +\frac{1}{4}\left[3 a \Phi_{m}^{2}-2 \delta\right]\left(\Phi_{m}-\Phi_{1}\right) \tanh (\hat{\xi}) \\
& \quad-\frac{3 a}{4} \Phi_{m}\left(\frac{1}{4} \tanh ^{3}(\xi)-\tanh ^{2}(\hat{\xi})\right)\left(\Phi_{m}-\Phi_{1}\right)^{2} \\
& +\frac{a}{4}\left(\frac{1}{5} \tanh ^{5}(\hat{\xi})-\frac{1}{4} \tanh ^{3}(\hat{\xi})-\tanh (\hat{\xi})\right) \\
& \left(\Phi_{m}-\Phi_{1}\right)^{3} .
\end{aligned}
$$

Hence, we have the following solution of Eq. (1):

$$
\begin{aligned}
A(x, t)= & i\left(\Phi_{m}+\left(\Phi_{1}-\Phi_{m}\right) \tanh ^{2}(\hat{\xi})\right)^{\frac{1}{2}} \\
& \exp [-i \Theta+i(\nu x-\lambda t),]
\end{aligned}
$$

where $\Theta(\hat{\xi})$ is given by $(64)$ and $\hat{\xi}=\tanh ^{-1} \sqrt{\frac{\Phi(\hat{\xi})-\Phi_{m}}{\Phi_{1}-\Phi_{m}}}$.
3.4 Consider case 4 in Sect. 2, for $b_{1}<0$ (see Fig. 5a-c)

(i) Corresponding to the level curves defined by $H(\phi, y)=0$ in (21), there exist two heteroclinic orbits of system (18) connecting the equilibrium point (cusp point) $E_{12}\left(r_{1}, 0\right)$ and the saddle equilibrium point $E_{3}\left(\Phi_{3}, 0\right)$ (see Fig. 5a). In this case, (23) can be written as

$$
\left(\sqrt{\frac{8 b_{5}}{15}}\right) \xi=\int_{\Phi_{0}}^{\Phi} \frac{\mathrm{d} \Phi}{\left(\Phi_{3}-\Phi\right)\left(\Phi-r_{1}\right)^{\frac{3}{2}}},
$$

where $\Phi_{0} \in\left(r_{1}, \Phi_{3}\right)$ and $r_{1}<0<\Phi_{3}$. Completing the above integral, we have the following parametric representation of $\Phi(\xi)$ :

$\Phi(\hat{\xi})=r_{1}+\left(r_{1}-\Phi_{3}\right) \tanh ^{2}(\hat{\xi})$,

we obtain from Eq. (10) that

$$
\begin{aligned}
& \Theta(\hat{\xi})=\left(\frac{1}{2}(2 v-\mu)+\frac{1}{2} \delta r_{1}+\frac{a}{4} r_{1}^{3}\right) \hat{\xi} \\
& +\frac{\hat{h}_{2}}{\Phi_{2}} \sqrt{\frac{r_{1}-\Phi_{3}}{r_{1}}} \tanh ^{-1}\left(\sqrt{\frac{r_{1}-\Phi_{3}}{r_{1}}} \tanh (\hat{\xi})\right) \\
& +\frac{1}{4}\left[3 a r_{1}^{2}-2 \delta\right]\left(\Phi_{3}-r_{1}\right) \tanh (\hat{\xi}) \\
& -\frac{3 a}{4} r_{1}\left(\frac{1}{4} \tanh ^{3}(\hat{\xi})-\tanh (\hat{\xi})\right)\left(\Phi_{3}-r_{1}\right)^{2} \\
& -\frac{a}{4}\left(\frac{1}{5} \tanh ^{5}(\xi)-\frac{1}{4} \tanh ^{3}(\hat{\xi})-\tanh (\hat{\xi})\right) \\
& \left(\Phi_{3}-r_{1}\right)^{3}
\end{aligned}
$$

where, $\hat{\xi}=\tanh ^{-1} \sqrt{\frac{\Phi(\hat{t})-r_{1}}{r_{1}-\Phi_{3}}}$. Hence, we have the following solution of Eq. (1):

$$
\begin{aligned}
A(x, t)= & i\left(r_{1}+\left(r_{1}-\Phi_{3}\right) \tanh ^{2}(\hat{\xi})\right) \\
& \exp [-i \Theta+i(\nu x-\lambda t),]
\end{aligned}
$$

where $\Theta(\hat{\xi})$ is given by (67).

(ii) Corresponding to a homoclinic orbit of system (18) to the saddle point $E_{3}\left(\Phi_{3}, 0\right)$ enclosing the origin $E_{0}(0,0)$ (see Fig. 5b). Hence, we obtain a similar parametric representation Eq. (1) as Eq. (65).

(iii) Corresponding to the curves defined by $\mathrm{H}_{2}(\Phi, y)=$ $h_{1}=h_{2}$ (see Fig. 5c), we have from Eq. (21) that $y^{2}=\omega_{5}\left(\Phi-r_{1}\right)^{3}\left(\Phi_{L}-\Phi\right)\left(\Phi_{M}-\Phi\right)$, where $r_{1}<\Phi_{3}<\Phi_{M}<0<\Phi_{L}$. Equation (18) has a homoclinic orbit to the cusp point $E_{12}\left(\Phi_{1}, 0\right)$. Then (23) has the form 


$$
=\int_{\Phi}^{\omega_{5} \xi} \frac{\mathrm{d} \Phi}{\left(\Phi-r_{1}\right) \sqrt{\left(\Phi-r_{1}\right)\left(\Phi_{M}-\Phi\right)\left(\Phi_{L}-\Phi\right)}} .
$$

It gives rise to the parametric representations of a homoclinic orbit of system (18) as follows:

$$
\Phi(\xi)=\Phi_{L}+\frac{\Phi_{M}-\Phi_{L}}{\operatorname{dn}^{2}\left(\omega_{5} \xi, k\right)} .
$$

Thus we obtain from (10) that

$$
\begin{aligned}
& \Theta(\xi)=\left(\frac{1}{2}(2 v-\mu)+\frac{1}{2} \delta \Phi_{L}+\frac{a}{4} \Phi_{L}^{3}\right) \xi \\
& +\frac{\hat{h}_{2}}{\Phi_{L}}\left(\frac{\Phi_{L}}{\Phi_{M}}-k^{2}\right) \Pi\left(\arcsin \left(\operatorname{sn}\left(\omega_{2} \xi, k\right)\right), \frac{\phi_{L}}{\Phi_{M}}, k\right) \\
& -\frac{1}{4}\left(2 \delta-3 a \Phi_{L}^{2}\right)\left(\Phi_{M}-\Phi_{L}\right) I_{4} \\
& \quad+\frac{a}{4}\left(3 \Phi_{L} I_{6}-\left(\Phi_{M}-\Phi_{L}\right) I_{5}\right)\left(\Phi_{M}-\Phi_{L}\right)^{2}
\end{aligned}
$$

Therefore, we have the following solution of Eq. (1):

$$
\begin{aligned}
A(x, t)= & i\left(\Phi_{L}+\frac{\Phi_{M}-\Phi_{L}}{\mathrm{dn}^{2}\left(\omega_{5} \xi, k\right)}\right)^{\frac{1}{2}} \\
& \times \exp [-i \Theta+i(\nu x-\lambda t)],
\end{aligned}
$$

where $\Theta(\xi)$ is given by (39), and $k^{2}=\frac{\Phi_{M}-r_{1}}{\Phi_{L}-r_{1}}$, $\omega_{5}=\frac{4 \sqrt{2 b_{5}}}{\sqrt{15\left(\Phi_{L}-r_{1}\right)}}, I_{4}=\frac{k^{2}}{k^{\prime 2}}\left[\beta_{4}-\operatorname{sn}\left(\omega_{5} \xi, k\right) \mathrm{cn}\right.$ $\left.\left(\omega_{2} \xi, k\right)\right], I_{5}=\frac{1}{5 k^{\prime 2}}\left(5-4 k^{2}\right) I_{6}, I_{6}=\frac{k^{2}}{k^{\prime 4}}\left[2\left(2-k^{2}\right)\right.$ $\beta_{4}-\operatorname{sn}\left(\omega_{2} \xi, k\right) \operatorname{cn}\left(\omega_{2} \xi, k\right)\left(k^{2}\left(\mathrm{nd}^{2}\left(\omega_{2} \xi, k\right)\right.\right.$ $-2)+4)]$.

\section{The bifurcations of phase portraits of system (18) when $p=3$}

In order to obtain the exact parametric representations of some orbits in the septic order nonlinearity of derivative of Schrödinger equation of system (18), we consider the case of $p=3$ with Hamiltonian given as:

$$
\begin{aligned}
H_{3}(\varphi, y)= & 3 \frac{y^{2}}{\varphi}-16 \varphi\left[b_{1}+\frac{1}{2} b_{3} \varphi\right. \\
& \left.+\frac{1}{3} b_{5} \varphi^{2}+\frac{1}{4} b_{7} \varphi^{3}\right] \equiv h .
\end{aligned}
$$

Obviously, if $\Delta=b_{3}^{2}-3 b_{2} b_{4}<0$, system (18) has only one equilibrium point $E_{0}(0,0)$. If $\Delta>$ $0, b_{7} \neq 0$, system (18) has three equilibrium points
$E_{0}(0,0), E_{1}\left(\varphi_{1}, 0\right)$ and $E_{2}\left(\varphi_{2}, 0\right)$, where $\varphi_{1,2}=$ $\frac{-b_{5} \pm \sqrt{\Delta}}{3 b_{7}}$. If $\Delta=0$, then $E_{1}$ and $E_{2}$ become a double equilibrium point.

Let $M\left(\varphi_{j}, 0\right)$ be the coefficient matrix of the linearized system of (18) at an equilibrium point $\left(\varphi_{j}, 0\right), j=1,2$. We have $J\left(\varphi_{j}, 0\right)=\operatorname{det} M\left(\varphi_{j}, 0\right)$ $=16 \varphi_{j}^{3}\left(b_{3}+2 b_{5} \varphi_{j}+3 b_{7} \varphi_{j}^{2}\right), j=1,2$ and $J(0,0)$ $=\operatorname{det} M(0,0)=0$.

We write for $H_{3}(\varphi, y)$ given by (9), $h_{0}=H_{3}(0,0)$, and $h_{j}=H_{3}\left(\varphi_{j}, 0\right)$. By using the above information to do qualitative analysis, we have the following bifurcations of phase portraits of system (18) shown in Fig. 8a-1.

\section{Explicit parametric representations of the solutions of system (18) when $p=3$}

In this section, we discuss the parametric representations of the solutions of system (18). Here, we take that $p=3$. We see from (72) and the first equation of (18) that

$$
\begin{aligned}
\frac{1}{\sqrt{3}} \xi & =\int_{\varphi_{0}}^{\varphi} \frac{\mathrm{d} \phi}{\sqrt{\varphi h+16 b_{1} \varphi^{2}+8 b_{3} \varphi^{3}+\frac{16}{3} b_{5} \varphi^{4}+4 b_{7} \varphi^{5}}} \\
& \equiv \sqrt{\frac{4 b_{7}}{3}} \int_{\varphi_{0}}^{\varphi} \frac{\mathrm{d} \varphi}{\sqrt{F_{5}(\varphi, h)}} .
\end{aligned}
$$

Thus, we shall find all possible exact explicit parametric representations for all bounded functions $\Phi=\sqrt{\varphi}$ where $\varphi>0$, determined by Eq. (18) in different parametric region of the $\left(b_{3}, b_{5}\right)$-parameter space.

5.1 The case of $\frac{2}{3} \sqrt{3 b_{3} b_{7}}<3 b_{1}^{\frac{3}{2}}, 0<h_{1}<h_{2}$ (see Fig. 8a)

For $h=h_{1}$, the level curve defined by $H_{3}(\Phi, y)=h$, Eq. (18) has a homoclinic orbit to the cusp point $E_{23}\left(\Phi_{2}, 0\right)$, enclosing the Equilibrium point $E_{1}\left(\Phi_{1}, 0\right)$. In this case $F_{5}=\left(\Phi-\Phi_{m}\right)\left(\Phi_{2}-\Phi\right)^{3} \Phi$, where $\Phi_{m}<\Phi_{1}<\Phi_{2}=\Phi_{3}<0$. Then Eq. (73) has the form

$\omega_{6} \xi=\int_{\Phi_{m}}^{\Phi} \frac{\mathrm{d} \Phi}{\left(\Phi_{2}-\Phi\right) \sqrt{\left(\Phi-\Phi_{m}\right)\left(\Phi_{2}-\Phi\right) \Phi}}$.

It gives rise to the parametric representations of a homoclinic orbit of system (18) as follows:

$\Phi(\xi)=\left(\Phi_{m}+\left(\Phi_{2}-\Phi_{m}\right) \operatorname{sn}^{2}\left(\omega_{6} \xi, k\right)\right)^{\frac{1}{2}}$. 


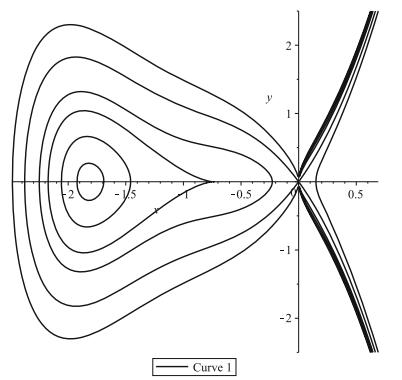

(a)

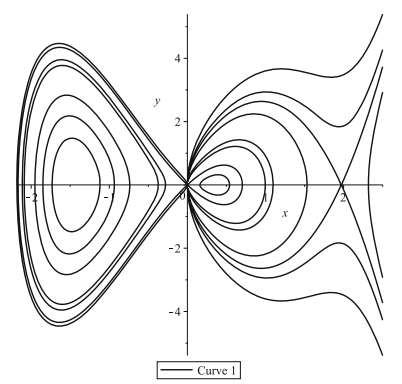

(d)

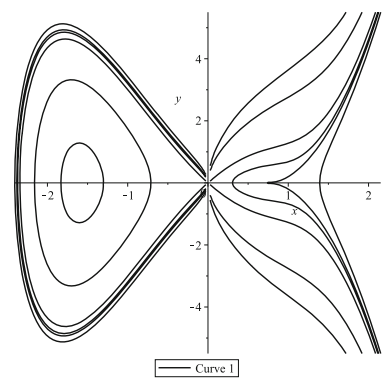

(g)

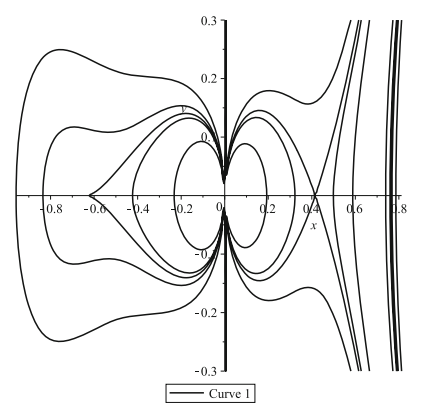

(i)

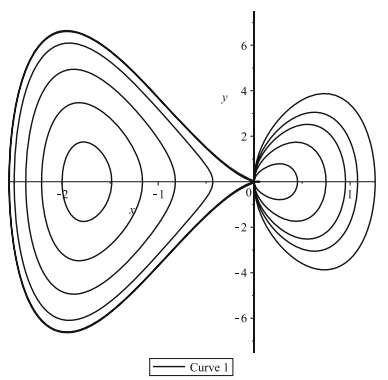

(b)

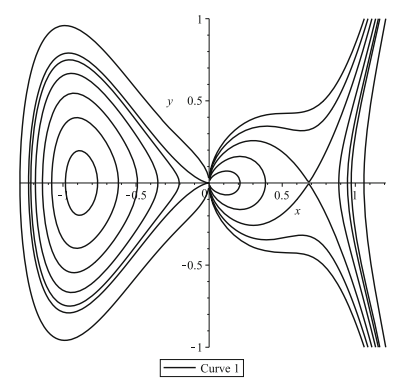

(c)

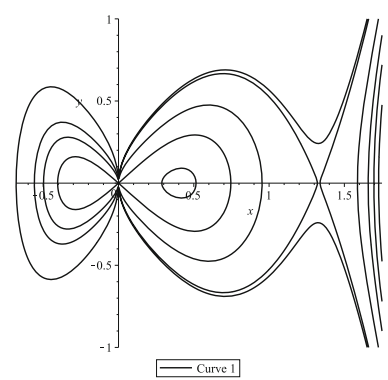

(e)

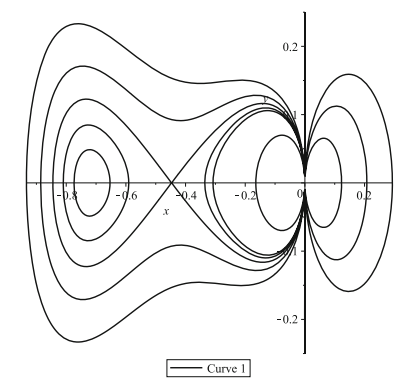

(h)

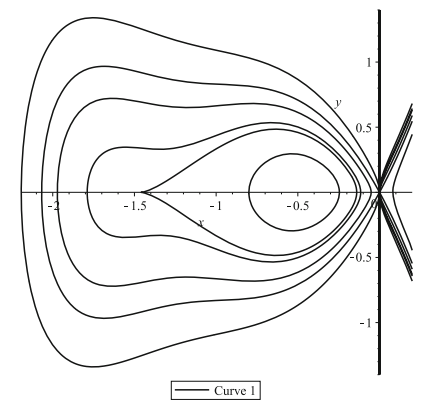

(k)

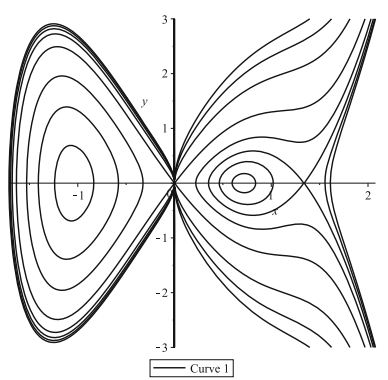

(f)

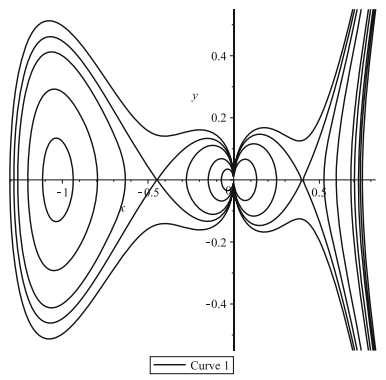

(i)

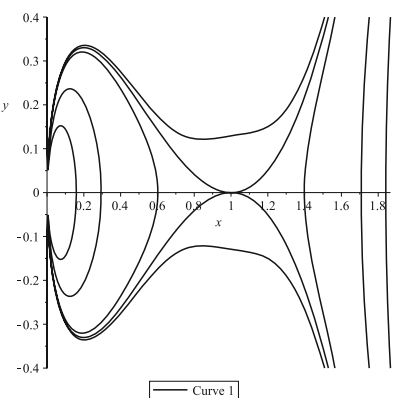

(l)
Fig. 8 Bifurcations of phase portraits of system (18), when $p=$ 3. a $\frac{2}{3} \sqrt{3 b_{3} b_{7}}<b_{5}, 0<h_{1}<h_{2}, \mathbf{b} b_{3}<2 \sqrt{b_{3} b_{7}} h_{1}<h_{2}$, c $\frac{2}{3} \sqrt{3 b_{3} b_{7}}<3 b_{1}^{\frac{3}{2}}, h_{1}<h_{2}, \mathbf{d} \frac{4}{3} \sqrt{3 b_{3} b_{7}}=2 \sqrt{b_{3} b_{7}}, 0<$ $h_{2}<h_{1}$, e $b_{3}<0<b_{7}, h_{1}<0<h_{2}$, f $-\frac{4}{3} \sqrt{3 b_{3} b_{7}}<$ $3 b_{1}^{\frac{3}{2}}<b_{5}, h_{2}<h_{1} \cdot \mathbf{g}-\frac{4}{3} \sqrt{-3 b_{3} b_{7}}<b 3<3 b_{1}^{\frac{3}{2}}, h_{2}<$ $0<h_{1}, \mathbf{h} 3 b_{1}^{\frac{3}{2}}<2 \sqrt{b_{3} b_{7}}, 0<h_{1}<h_{2}$, $\mathbf{i}\left(b_{3}, b_{5}\right)=$ $\left(3 b_{1}^{\frac{3}{2}},-3 b_{1}^{\frac{3}{2}}\right), h_{2}<h_{1}, \mathbf{j}-3 b_{1}^{\frac{3}{2}}<b_{5}<3 b_{1}^{\frac{3}{2}}, h_{2}=h_{1}, \mathbf{k}$ $3 b_{1}^{\frac{3}{2}}<2 \sqrt{b_{3} b_{7}}, 0<h_{1}<h_{2}, \mathbf{l} b_{3}<0<b_{5}, \Delta=0, h_{1}=h_{2}$ 
Thus, we obtain from (10), that

$$
\begin{aligned}
\Theta(\xi)= & \frac{1}{2}(2 v-\mu) \xi+\frac{1}{2} \delta \int_{\Phi_{m}}^{\Phi}\left(\Phi_{m}+\left(\Phi_{2}-\Phi_{m}\right)\right. \\
& \left.\times \operatorname{sn}^{2}\left(\omega_{6} \xi, k\right)\right)^{\frac{1}{2}} \mathrm{~d} \xi \\
& +\hat{h}_{2} \int_{\Phi_{m}}^{\Phi}\left(\frac{1}{\Phi_{m}+\left(\Phi_{2}-\Phi_{m}\right) \operatorname{sn}^{2}\left(\omega_{6} \xi, k\right)}\right)^{\frac{1}{2}} \mathrm{~d} \xi \\
& +\frac{a}{4} \int_{\Phi_{m}}^{\Phi}\left(\Phi_{m}+\left(\Phi_{2}-\Phi_{m}\right) \operatorname{sn}^{2}\left(\omega_{6} \xi, k\right)\right)^{\frac{3}{2}} \mathrm{~d} \xi .
\end{aligned}
$$

Therefore, we have the following solution of Eq. (1):

$$
\begin{aligned}
A(x, t)= & i\left(\Phi_{m}+\left(\Phi_{2}-\Phi_{m}\right) \operatorname{sn}^{2}\left(\omega_{6} \xi, k\right)\right)^{\frac{1}{2}} \\
& \times \exp [-i \Theta+i(v x-\lambda t),]
\end{aligned}
$$

where $\Theta(\xi)$ is given by (75), and $k^{2}=\frac{\Phi_{2}-\Phi_{m}}{\left|\Phi_{m}\right|}, \omega_{6}=$ $4 \sqrt{\frac{b_{7}}{3\left|\Phi_{m}\right|}}$.

5.2 The case of $-3 b_{1}^{\frac{3}{2}}<\sqrt{\left|b_{3} b_{7}\right|} h_{1}<h_{2}$ (see Fig. 8c).

(i) For $h \in\left(h_{1}, h_{2}\right)$ the level curves defined by $H_{3}(\Phi, y)=h$ in (72), there exist a periodic orbits of system (18) enclosing an equilibrium point of $E_{1}\left(\Phi_{1}, 0\right)$. In this case we have $F_{5}=\left(\Phi-r_{2}\right)\left(r_{1}-\right.$ $\Phi) \Phi\left(\Phi_{3}-\Phi\right)^{2}$, where $r_{2}<\Phi_{1}<r_{1}<0<\Phi_{2}<$ $\Phi_{3}$. Now, (73) can be written as

$\omega_{7} \xi=\int_{r_{2}}^{\Phi} \frac{\mathrm{d} \Phi}{\left(\Phi_{3}-\Phi\right) \sqrt{\left(\Phi-r_{2}\right)\left(r_{1}-\Phi\right) \Phi}}$.

Completing the above integral, we can get a periodic orbit of Eq. (1):

$\Phi(\xi)=\left(r_{1}+\left(r_{2}-r_{1}\right) \operatorname{sn}^{2}\left(\omega_{7} \xi, k\right)\right)^{\frac{1}{2}}$,

we obtain from Eq. (10) that

$$
\begin{aligned}
\Theta(\xi)= & \frac{1}{2}(2 v-\mu) \xi+\frac{1}{2} \delta \int_{r_{2}}^{\Phi}\left(r_{1}+\left(r_{2}-r_{1}\right)\right. \\
& \left.\operatorname{sn}^{2}\left(\omega_{7} \xi, k\right)\right)^{\frac{1}{2}} \mathrm{~d} \xi
\end{aligned}
$$

$$
\begin{aligned}
& +\hat{h}_{2} \int_{r_{2}}^{\Phi}\left(\frac{1}{r_{1}+\left(r_{2}-r_{1}\right) \operatorname{sn}^{2}\left(\omega_{7} \xi, k\right)}\right)^{\frac{1}{2}} \mathrm{~d} \xi \\
& +\frac{a}{4} \int_{r_{2}}^{\Phi}\left(r_{1}+\left(r_{2}-r_{1}\right) \operatorname{sn}^{2}\left(\omega_{7} \xi, k\right)\right)^{\frac{3}{2}} \mathrm{~d} \xi .
\end{aligned}
$$

Hence, from Eqs. (77) and (78) we have the following solution of Eq. (1):

$$
\begin{aligned}
A(x, t)= & i\left(r_{1}+\left(r_{2}-r_{1}\right) \operatorname{sn}^{2}\left(\omega_{7} \xi, k\right)\right)^{\frac{1}{4}} \\
& \times \exp [-i \Theta+i(\nu x-\lambda t)],
\end{aligned}
$$

where $k^{2}=\left|\frac{r_{2}-r_{1}}{r_{2}}\right|, \omega_{7}=\frac{4 \sqrt{b_{7}}}{\sqrt{3\left|r_{2}\right|}}$.

(ii) Corresponding to the level curves defined by $H_{3}(\Phi, y)=h, h \in\left(h_{1}, h_{2}\right)$ in (72), there exist a homoclinic orbits of system (18) at the equilibrium points $E_{0}(0,0)$, enclosing the equilibrium point $E_{2}\left(\Phi_{2}, 0\right)$. Now, (73) can be written as

$$
\omega_{7} \xi=\int_{0}^{\Phi} \frac{\mathrm{d} \Phi}{\left(\Phi_{3}-\Phi\right) \sqrt{\left(\Phi-r_{2}\right)\left(\Phi-r_{1}\right) \Phi}} .
$$

Completing the above integral, we have the following parametric representation of Eq. (1):

$$
\Phi(\xi)=\sqrt{r_{1}}\left(1-\frac{1}{1-\operatorname{sn}^{2}\left(\omega_{7} \xi, k\right)}\right)^{\frac{1}{2}},
$$

we obtain from Eq. (10) that

$$
\begin{aligned}
\Theta(\xi)= & \frac{1}{2}(2 v-\mu) \xi+\frac{\delta}{2} \sqrt{\left|r_{1}\right|} \int_{0}^{\Phi} \\
& \left(1-\frac{1}{1-\mathrm{sn}^{2}\left(\omega_{7} \xi, k\right)}\right)^{\frac{1}{2}} \mathrm{~d} \xi \\
& +\frac{\hat{h}_{2}}{\sqrt{r_{1}}} \int_{0}^{\Phi}\left(1-\frac{1}{1-\mathrm{sn}^{2}\left(\omega_{7} \xi, k\right)}\right)^{-\frac{1}{2}} \mathrm{~d} \xi \\
& +\frac{a}{4} \int_{0}^{\Phi}\left[r_{1}\left(1-\frac{1}{1-\mathrm{sn}^{2}\left(\omega_{7} \xi, k\right)}\right)\right]^{\frac{3}{2}} \mathrm{~d} \xi .
\end{aligned}
$$

Hence, we have the following solution of Eq. (1):

$$
\begin{aligned}
A(x, t)= & i\left[r_{1}\left(1-\frac{1}{1-\mathrm{sn}^{2}\left(\omega_{7} \xi, k\right)}\right)\right]^{\frac{1}{4}} \\
& \times \exp [-i \Theta+i(\nu x-\lambda t)],
\end{aligned}
$$

where $\Theta(\xi)$ is given by (81) and $\Phi_{3} \neg 0$.

(iii) For $h=0$, the level curves defined by $H_{2}(\Phi, y)=$ $h$, Eq. (18) has a homoclinic orbit to the origin (cusp point) $E_{0}(0,0)$ enclosing an equilibrium point of $E_{1}\left(\Phi_{1}, 0\right)$. In this case we have $F_{5}=\left(\Phi_{m}-\right.$ 
$\Phi) \Phi^{3}\left(\Phi_{L}-\Phi\right)$, where $\Phi_{m}<\Phi_{1}<0<\Phi_{L}$. Now, (73) can be written as

$$
\omega_{8} \xi=\int_{\Phi_{m}}^{\Phi} \frac{\mathrm{d} \Phi}{\Phi \sqrt{\left(\Phi_{m}-\Phi\right) \Phi\left(\Phi_{L}-\Phi\right)}} .
$$

Completing the above integral, we can get a periodic orbit of Eq. (1):

$$
\Phi(\xi)=\left(\Phi_{m}\left(1-\operatorname{sn}^{2}\left(\omega_{8} \xi, k\right)\right)\right)^{\frac{1}{2}},
$$

we obtain from Eq. (10) that

$$
\begin{aligned}
\Theta(\xi)= & \frac{1}{2}(2 v-\mu) \xi+\frac{\delta}{2} \sqrt{\Phi_{m}} \int_{\Phi_{m}}^{\Phi} \\
& \left(\left(1-\operatorname{sn}^{2}\left(\omega_{8} \xi, k\right)\right)\right)^{\frac{1}{2}} \mathrm{~d} \xi \\
& +\frac{\hat{h}_{2}}{\sqrt{\Phi_{m}}} \int_{\Phi_{m}}^{\Phi}\left(\frac{1}{\left(1-\mathrm{sn}^{2}\left(\omega_{8} \xi, k\right)\right)}\right)^{\frac{1}{2}} \mathrm{~d} \xi \\
& +\frac{a}{4} \int_{\Phi_{m}}^{\Phi}\left(\Phi_{m}\left(1-\mathrm{sn}^{2}\left(\omega_{8} \xi, k\right)\right)\right)^{\frac{3}{2}} \mathrm{~d} \xi
\end{aligned}
$$

Hence, we have the following solution of Eq. (1):

$$
\begin{aligned}
A(x, t)= & i\left(\Phi_{m}\left(1-\operatorname{sn}^{2}\left(\omega_{8} \xi, k\right)\right)\right)^{\frac{1}{4}} \\
& \times \exp [-i \Theta+i(v x-\lambda t)]
\end{aligned}
$$

where $\Theta(\xi)$ is given by (84) and $k^{2}=\frac{\mid \Phi_{m}}{\Phi_{L}-\Phi_{m}}, \omega_{8}$ $=\frac{4 \sqrt{b_{7}}}{\sqrt{3\left(\Phi_{L}-\Phi_{m}\right)}}$.

5.3 The case of $b_{3}<0<b_{5}, h_{1}<0<h_{2}$ (see

Fig. 8d)

For $h=0$, the level curve defined by $H_{3}(\Phi, y)=h$, there exist two homoclinic orbit to the origin $E_{0}(0,0)$ with "figure eight" of (18). In this case we have $F_{5}=$ $\left(\Phi-\Phi_{m}\right) \Phi^{2}\left(\Phi_{M}-\Phi\right)\left(\Phi_{L}-\Phi\right)$, where $\Phi_{m}<\Phi_{1}<$ $0<\Phi_{2}<\Phi_{M}<\Phi_{L}$. Now, (73) has the form

$\omega_{8} \xi=\int_{\Phi}^{\Phi_{M}} \frac{\mathrm{d} \Phi}{\Phi \sqrt{\left(\Phi-\Phi_{m}\right)\left(\Phi_{M}-\Phi\right)\left(\Phi_{L}-\Phi\right)}}$

and

$\omega_{8} \xi=\int_{\Phi_{m}}^{\Phi} \frac{\mathrm{d} \Phi}{\Phi \sqrt{\left(\Phi-\Phi_{m}\right)\left(\Phi_{M}-\Phi\right)\left(\Phi_{L}-\Phi\right)}}$.

Using the above integrals, corresponding to the right homoclinic orbit we have the following parametric representation of Eq. (1):

$\Phi(\xi)=\left(\Phi_{L}+\frac{\Phi_{M}-\Phi_{L}}{\operatorname{dn}^{2}\left(\omega_{8} \xi, k\right)}\right)^{\frac{1}{2}}$, we obtain from Eq. (10) that

$$
\begin{aligned}
\Theta(\xi)= & \frac{1}{2}(2 v-\mu) \xi \\
& +\frac{1}{2} \delta \int_{\Phi}^{\Phi_{M}}\left(\Phi_{L}+\frac{\Phi_{M}-\Phi_{L}}{\mathrm{dn}^{2}\left(\omega_{8} \xi, k\right)}\right)^{\frac{1}{2}} \mathrm{~d} \xi \\
& +\hat{h}_{2} \int_{\Phi}^{\Phi_{M}}\left(\Phi_{L}+\frac{\Phi_{M}-\Phi_{L}}{\mathrm{dn}^{2}\left(\omega_{8} \xi, k\right)}\right)^{-\frac{1}{2}} \mathrm{~d} \xi \\
& +\frac{a}{4} \int_{\Phi}^{\Phi_{M}}\left(\Phi_{L}+\frac{\Phi_{M}-\Phi_{L}}{\mathrm{dn}^{2}\left(\omega_{8} \xi, k\right)}\right)^{\frac{3}{2}} \mathrm{~d} \xi
\end{aligned}
$$

Hence, we have the following solution of Eq. (1):

$$
\begin{aligned}
A(x, t)= & i\left(\Phi_{L}+\frac{\left(\Phi_{M}-\Phi_{L}\right)}{\mathrm{dn}^{2}\left(\omega_{8} \xi, k\right)}\right)^{\frac{1}{4}} \\
& \times \exp [-i \Theta+i(\nu x-\lambda t)],
\end{aligned}
$$

where $\Theta(\xi)$ is given by (87) and corresponding to the left homoclinic orbit, we have the following parametric representation of Eq. (1):

$\Phi(\xi)=\left(\Phi_{m}+\left(\Phi_{M}-\Phi_{m}\right) \operatorname{sn}^{2}\left(\omega_{8} \xi, k\right)\right)^{\frac{1}{2}}$.

Thus we obtain from Eq. (10) that

$$
\begin{aligned}
& \Theta(\xi)=\frac{1}{2}(2 v-\mu) \xi \\
& +\frac{1}{2} \delta \int_{\Phi_{m}}^{\Phi}\left(\Phi_{m}+\left(\Phi_{M}-\Phi_{m}\right) \operatorname{sn}^{2}\left(\omega_{8} \xi, k\right)\right)^{\frac{1}{2}} \mathrm{~d} \xi \\
& +\hat{h}_{2} \int_{\Phi_{m}}^{\Phi}\left(\Phi_{m}+\left(\Phi_{M}-\Phi_{m}\right) \operatorname{sn}^{2}\left(\omega_{8} \xi, k\right)\right)^{-\frac{1}{2}} \mathrm{~d} \xi \\
& +\frac{a}{4} \int_{\Phi_{m}}^{\Phi}\left(\Phi_{m}+\left(\Phi_{M}-\Phi_{m}\right) \mathrm{sn}^{2}\left(\omega_{8} \xi, k\right)\right)^{\frac{3}{2}} \mathrm{~d} \xi
\end{aligned}
$$

Hence, we have the following solution of Eq. (1):

$$
\begin{aligned}
A(x, t)= & i\left(\Phi_{m}+\left(\Phi_{M}-\Phi_{m}\right) \operatorname{sn}^{2}\left(\omega_{8} \xi, k\right)\right)^{\frac{1}{4}} \\
& \times \exp [-i \Theta+i(\nu x-\lambda t)],
\end{aligned}
$$

where $\Theta(\xi)$ is given by (90) and $k^{2}=\frac{\Phi_{M}-\Phi_{m}}{\Phi_{L}-\Phi_{m}}$.

5.4 The case of $-\frac{4}{3} \sqrt{-3 b_{3} b_{7}}<b 3<3 b_{1}^{\frac{3}{2}}, h_{2}<0<h_{1}$ (see Fig. 8g)

For $h=h_{1}$ the curves defined by $H_{2}(\Phi, y)=h$, we have $y^{2}=\left(\frac{8 b_{5}}{15}\right)\left(\Phi-r_{1}\right) \Phi\left(\Phi_{2}-\Phi\right)^{3}$, where 
$r_{1}<\Phi_{1}<0<\Phi_{2}=\Phi_{3}$. Equation (18) has a periodic orbit enclosing $E_{1}\left(\Phi_{1}, 0\right)$, which gives rise to a periodic orbit of system (18). Now, (23) has the form $\omega_{4} \xi=\int_{r_{1}}^{\Phi} \frac{\mathrm{d} \Phi}{\left(\Phi_{2}-\Phi\right) \sqrt{\left(\Phi-r_{1}\right) \Phi\left(\Phi_{2}-\Phi\right)}}$.

Therefore, we obtain the following parametric representation:

$\Phi(\xi)=\sqrt{r_{1}}\left(1-\operatorname{sn}^{2}\left(\omega_{4} \xi, k\right)\right)^{\frac{1}{2}}$,

Thus we obtain from (10) that

$$
\begin{aligned}
\Theta(\xi)= & \frac{1}{2}(2 v-\mu) \xi+\frac{\delta}{2} \sqrt{r_{1}} \\
& \int_{r_{1}}^{\Phi} \sqrt{1-\mathrm{sn}^{2}\left(\omega_{4} \xi, k\right)} \mathrm{d} \xi \\
& +\frac{\hat{h}_{2}}{\sqrt{r_{1}}} \int_{r_{1}}^{\Phi} \frac{\mathrm{d} \xi}{\sqrt{1-\mathrm{sn}^{2}\left(\omega_{4} \xi, k\right)}} \\
& +\frac{a}{4} \int_{r_{1}}^{\Phi}\left(r_{1}\left(1-\mathrm{sn}^{2}\left(\omega_{4} \xi, k\right)\right)\right)^{\frac{3}{2}} \mathrm{~d} \xi .
\end{aligned}
$$

Hence, we have the following solution of Eq. (1):

$$
\begin{aligned}
A(x, t)= & i \sqrt{r_{1}\left(1-\operatorname{sn}^{2}\left(\omega_{4} \xi, k\right)\right)} \\
& \times \exp [-i \Theta+i(\nu x-\lambda t)],
\end{aligned}
$$

where $\Theta(\xi)$ is given by (93) and $k^{2}=\frac{\left|r_{1}\right|}{\Phi_{2}-r_{2}}$.

5.5 The case of $3 b_{1}^{\frac{3}{2}}<2 \sqrt{b_{3} b_{7}}, 0<h_{1}<h_{2}$ (see

Fig. 8h)

For $h \in\left(h_{1}, 0\right)$, the level curve defined by $H_{3}(\Phi, y)=$ $h$, there exist two homoclinic orbit to the equilibrium point $E_{2}\left(\Phi_{2}, 0\right)$ of system (18). Here, the origin is $E_{0}(0,0)$ is a high order equilibrium point. In this case we have $F_{5}=\left(\Phi-\Phi_{m}\right)\left(\Phi-\Phi_{2}\right)^{2} \Phi\left(\Phi_{L}-\Phi\right)$, where $\Phi_{m}<\Phi_{1}<\Phi_{2}<\Phi_{3}<0<\Phi_{L}$. Now, (73) has the form

$\omega_{8} \xi=\int_{\Phi_{m}}^{\Phi} \frac{\mathrm{d} \Phi}{\left(\Phi_{2}-\Phi\right) \sqrt{\left(\Phi-\Phi_{m}\right) \Phi\left(\Phi_{L}-\Phi\right)}}$

and

$\omega_{8} \xi=\int_{\Phi}^{0} \frac{\mathrm{d} \Phi}{\left(\Phi-\Phi_{2}\right) \sqrt{\left(\Phi-\Phi_{m}\right) \Phi\left(\Phi_{L}-\Phi\right)}}$.

Using the above integrals, corresponding to the left homoclinic orbit we have the following parametric representation of Eq. (1):

$\Phi(\xi)=\left(\Phi_{m}\left(1-\operatorname{sn}^{2}\left(\omega_{8} \xi, k\right)\right)\right)^{\frac{1}{2}}$, we obtain from Eq. (10) that

$$
\begin{aligned}
\Theta(\xi)= & \frac{1}{2}(2 v-\mu) \xi \\
& +\frac{\delta}{2} \sqrt{\left|\Phi_{m}\right|} \int_{\Phi_{m}}^{\Phi}\left(1-\operatorname{sn}^{2}\left(\omega_{8} \xi, k\right)\right)^{\frac{1}{2}} \mathrm{~d} \xi \\
& +\frac{\hat{h}_{2}}{\sqrt{\left|\Phi_{m}\right|}} \int_{\Phi_{m}}^{\Phi}\left(1-\operatorname{sn}^{2}\left(\omega_{8} \xi, k\right)\right)^{-\frac{1}{2}} \mathrm{~d} \xi \\
& +\frac{a}{4} \int_{\Phi_{m}}^{\Phi}\left(\Phi_{m}\left(1-\operatorname{sn}^{2}\left(\omega_{8} \xi, k\right)\right)\right)^{\frac{3}{2}} \mathrm{~d} \xi .
\end{aligned}
$$

Hence, we have the following solution of Eq. (1):

$$
\begin{aligned}
A(x, t)= & i\left(\Phi_{m}\left(1-\mathrm{sn}^{2}\left(\omega_{8} \xi, k\right)\right)\right)^{\frac{1}{4}} \\
& \times \exp [-i \Theta+i(\nu x-\lambda t)],
\end{aligned}
$$

where $\Theta(\xi)$ is given by (96) and $k^{2}=\frac{\left|\Phi_{m}\right|}{\Phi_{L}-\Phi_{m}}, \Phi_{m} \neq$ $\Phi_{2}$.

and corresponding to the right homoclinic orbit, we have the following parametric representation of Eq. (1):

$\Phi(\xi)=\left(\Phi_{L}\left(1-\frac{1}{\mathrm{dn}^{2}\left(\omega_{8} \xi, k\right)}\right)\right)^{\frac{1}{2}}$,

we obtain from Eq. (10) that

$$
\begin{aligned}
\Theta(\xi)= & \frac{1}{2}(2 v-\mu) \xi \\
& +\frac{\delta}{2} \sqrt{\Phi_{L}} \int_{\Phi}^{0}\left(1-\frac{1}{\mathrm{dn}^{2}\left(\omega_{8} \xi, k\right)}\right)^{\frac{1}{2}} \mathrm{~d} \xi \\
& +\frac{\hat{h}_{2}}{\sqrt{\Phi_{L}}} \int_{\Phi}^{\Phi_{M}}\left(1-\frac{1}{\mathrm{dn}^{2}\left(\omega_{8} \xi, k\right)}\right)^{-\frac{1}{2}} \mathrm{~d} \xi \\
& +\frac{a}{4} \int_{\Phi}^{\Phi_{M}}\left(\Phi_{L}\left(1-\frac{1}{\mathrm{dn}^{2}\left(\omega_{8} \xi, k\right)}\right)^{\frac{3}{2}} \mathrm{~d} \xi .\right.
\end{aligned}
$$

Hence, we have the following solution of Eq. (1):

$$
\begin{aligned}
A(x, t)= & i\left(\Phi_{L}\left(1-\frac{1}{\operatorname{dn}^{2}\left(\omega_{8} \xi, k\right)}\right)^{\frac{1}{4}}\right. \\
& \times \exp [-i \Theta+i(\nu x-\lambda t)],
\end{aligned}
$$

where $\Theta(\xi)$ is given by (99).

5.6 The case of $3 b_{1}^{\frac{3}{2}}<2 \sqrt{b_{3} b_{7}}, 0<h_{1}<h_{2}$ (see

Fig. 8k).

For $h=h_{2}$, the level curves defined by $H(\Phi, y)=h$, in (72), we have a homoclinic orbit at a cusp point $E_{12}\left(\Phi_{1}, 0\right)$ of system $(18)$ enclosing $E_{3}\left(\Phi_{3}, 0\right)$. In this 
case we have $F_{5}=\left(\Phi-\Phi_{1}\right)^{3}\left(\Phi_{M}-\Phi\right) \Phi$, where $\Phi_{1}<\Phi_{3}<\Phi_{M}<0$. Now, (73) can be written as

$\omega_{6} \xi=\int_{\Phi}^{\Phi_{M}} \frac{\mathrm{d} \Phi}{\left(\Phi-\Phi_{1}\right) \sqrt{\left(\Phi-\Phi_{1}\right)\left(\Phi_{M}-\Phi\right) \Phi}}$.

It gives rise to the parametric representations of Eq. (1) as follows:

$\Phi(\xi)=\sqrt{\Phi_{M}} \operatorname{nd}\left(\omega_{6} \xi, k\right)$,

Thus we obtain from (10) that

$$
\begin{aligned}
& \Theta(\xi)=\frac{1}{2}(2 v-\mu) \xi \\
& +\frac{\delta \sqrt{\Phi_{M}}}{2 k^{\prime}} \tanh ^{-1}\left[\frac{k^{\prime} \operatorname{sn}\left(\omega_{6} \xi, k\right)-\operatorname{cn}\left(\omega_{2} \xi, k\right)}{k^{\prime} \operatorname{sn}\left(\omega_{6} \xi, k\right)+\operatorname{cn}\left(\omega_{2} \xi, k\right)}\right] \\
& +\frac{\hat{h}_{2}}{\sqrt{\Phi_{M}}} \sinh ^{-1}\left(\omega_{2} \xi, k\right)+\frac{a}{4 k^{\prime 3}} \Phi_{M}^{\frac{3}{2}} \\
& \quad\left(\left(2-k^{2}\right) \tanh ^{-1}\left(\frac{k^{\prime} \operatorname{sn}\left(\omega_{6} \xi, k\right)-\operatorname{cn}\left(\omega_{2} \xi, k\right)}{k^{\prime} \operatorname{sn}\left(\omega_{6} \xi, k\right)+\operatorname{cn}\left(\omega_{2} \xi, k\right)}\right)\right) \\
& -\frac{a}{4 k^{3}} \Phi_{M}^{\frac{3}{2}} k^{\prime} k^{2} \operatorname{sn}\left(\omega_{2} \xi, k\right) \operatorname{cn}\left(\omega_{2} \xi, k\right) \operatorname{nd}^{2}\left(\omega_{2} \xi, k\right) .
\end{aligned}
$$

Therefore, we have the following solution of Eq. (1):

$$
\begin{aligned}
A(x, t)= & i\left(\Phi_{M} \mathrm{nd}^{2}\left(\omega_{6} \xi, k\right)\right)^{\frac{1}{4}} \\
& \times \exp [-i \Theta+i(\nu x-\lambda t)],
\end{aligned}
$$

where $\Theta(\xi)$ is given by (102).

5.7 The case of $b_{3}<0<b_{5}, \Delta=0, h_{1}=h_{2}$ (see

Fig. 81).

For $h=h_{1}$, the level curves defined by $H(\Phi, y)=h$, in (72), we have a homoclinic orbit to the multiple equilibrium point (cusp point) $\left(\Phi_{1}, 0\right)$ and $\Phi_{1}=\Phi_{2}$ of system (18) enclosing $E_{3}\left(\Phi_{3}, 0\right)$. In this case we have $F_{5}=\left(\Phi-\Phi_{1}\right)^{4} \Phi$, where $b_{2}=3\left(b_{1}\right)^{\frac{3}{2}}, b_{3}=$ $-3\left(b_{1}\right)^{\frac{3}{2}}$ and $\Phi_{1}<\Phi_{3}<\Phi_{M}<0$. Now, (73) can be written as

$\omega_{6} \xi=\int_{0}^{\Phi} \frac{\mathrm{d} \Phi}{\left(\Phi_{2}-\Phi\right)^{2} \sqrt{\Phi}}$.

It gives rise to the parametric representations of Eq. (1) as follows:

$\Phi(\hat{\xi})=\sqrt{\Phi_{2}} \tanh (\hat{\xi})$, and by using Eq. (10) we have

$$
\begin{aligned}
\Theta((\hat{\xi}))= & \frac{1}{2}(2 v-\mu) \hat{\xi}+\frac{\delta}{2} \sqrt{\Phi_{2}} \ln (\cosh (\hat{\xi})) \\
& +\frac{1}{2} \hat{h}_{2} \ln \left(\frac{1+\sinh (\hat{\xi})}{1-\sinh (\hat{\xi})}\right)+\frac{a}{4} \Phi_{2}^{\frac{3}{2}} \frac{\sinh (\hat{\xi})}{2 \cosh ^{2}(\hat{\xi})} \\
& +\frac{1}{4} \ln \left(\frac{1+\sinh (\hat{\xi})}{1-\sinh (\hat{\xi})}\right) .
\end{aligned}
$$

Hence, we have the following solution of Eq. (1):

$$
\begin{aligned}
A(x, t)= & i\left(\Phi_{2} \tanh ^{2}(\hat{\xi})\right)^{\frac{1}{4}} \\
& \times \exp [-i \Theta+i(v x-\lambda t)],
\end{aligned}
$$

where $\Theta(\hat{\xi})$ is given by (105).

\section{Conclusion}

To sum up, we have proved the following Theorems.

Theorem 1 Depending on the changes of system parameters, the bifurcations of phase portraits of system (18) are shown in Figs. 1, 2, 3, 4, 5 and 6

(i) Depending on change of parameters regions of $\left(b_{2}, b_{3}, b_{4}\right)$ for $b_{1}<0$ and $b_{1}>0$, we have found 18 solutions corresponding to the periodic, homoclinic and heteroclinic orbits of system (18) with $p=2$. The septic derivative nonlinear Schrödinger equation (1) has 18 exact solutions given by (26), (29), (32), (35), (38), (41), (44), (47), (50), (53), (56), (59), (62), (65), (68), and (71).

(ii) System (3) has 18 exact explicit solutions $\Phi(\xi)=$ $\sqrt{\Phi} \sin \Theta$, and $\psi(\xi)=\sqrt{\Phi} \cos \Theta$, where $\Phi(\xi)$ and $\psi(\xi)$ are given in Sect. 3 .

Theorem 2 (i) Depending on change of parameters regions of $\left(b_{2}, b_{3}, b_{4}\right)$ for $b_{1}<0$ and $b_{1}>0$, we have found 11 solutions corresponding to the periodic, homoclinic and heteroclinic orbits of system (18) with $p=3$. The septic derivative nonlinear Schrödinger equation (1) has 11 exact solutions given by (76), (79), (82), (85), (88), (91), (94), (97), (100), (103) and (106).

(ii) System (3) has 11 exact explicit solutions $\Phi(\xi)=$ $\sqrt{\Phi} \sin \Theta$, and $\psi(\xi)=\sqrt{\Phi} \cos \Theta$, where $\Phi(\xi)$ and $\psi(\xi)$ are given in Sect. 4. 
Open Access This article is distributed under the terms of the Creative Commons Attribution 4.0 International License (http://creativecommons.org/licenses/by/4.0/), which permits unrestricted use, distribution, and reproduction in any medium, provided you give appropriate credit to the original author(s) and the source, provide a link to the Creative Commons license, and indicate if changes were made.

\section{References}

1. Agrawal, G.P., Kivshar, Y.S.: Optical Solitons: From Fibers to Photonic Crystals. Academic Press, San Diego (2003)

2. Marklund, M., Shukla, P.K., Stenflo, L.: Ultrashort solitons and kinetic effects in nonlinear metamaterials. Phys. Rev. E 73, 037601 (2006)

3. Dalfovo, F.: Theory of Bose-Einstein condensation in trapped gases. Rev. Mod. Phys. 71(3), 463-512 (1999)

4. Birnbaum, Z., Malomed, B.A.: Families of spatial solitons in a two-channel waveguide with the cubic-quintic nonlinearity. Phys. D 237, 3252-3262 (2008)

5. Karpman, I.V., Shagalov, A.G.: Solitons and their stability in high dispersive systems. I. Fourth-order nonlinear Schrödinger-type equations with power-law nonlinearities. Phys. Lett. A 228, 59-65 (1997)

6. Peleg, A., Chung, Y., Dohnal, T., Nguyen, Q.M.: Diverging probability density functions for flat-top solitary waves. Phys. Rev. E 80, 026602 (2009)

7. Caradoc-Davies, B.M., Ballagh, R.J., Burnett, K.: Coherent dynamics of vortex formation in trapped Bose-Einstein condensates. Phys. Rev. Lett. 83(5), 895-898 (1999)

8. Pushkarov, KhI, Pushkarov, D.I., Tomov, I.V.: Self-action of light beams in nonlinear media: soliton solutions. Opt. Quantum Electron. 11, 471-478 (1979)

9. Chow, K., Rogers, C.: Localized and periodic wave patterns for a nonic nonlinear Schrödinger equation. Phys. Lett. A 377, 2546-2550 (2013)

10. Wamba, E.M., Ekogo, T.B., Atangana, J., Kofane, T.C.: Effects of threebody interactions in the parametric and modulational instabilities of Bose-Einstein condensates. Phys. Lett. A 375, 4288-4295 (2011)

11. Guckenheimer, J., Holmes, P.J.: Nonlinear Oscillations, Dynamical Systems and Bifurcations of Vector Fields. Springer, Berlin (1983)
12. Chow, K.W.: Periodic waves for a system of coupled, higher order nonlinear Schrödinger equations with third order dispersion. Phys. Lett. A 308(5-6), 426-431 (2003)

13. Rogers, C., Chow, K.: Localized pulses for the quintic derivative nonlinear Schrödinger equation on a continuouswave background. Phys. Rev. E 86, 037601 (2012)

14. Rogers, C., Malomed, B., Li, H., Chow, K.: Propagating wave patterns in a derivative nonlinear Schrödinger system with quintic nonlinearity. J. Phys. Soc. Jpn. 81, 094005 (2012a)

15. Rogers, C., Malomed, B., Chow, K.: Invariants in a resonant nonlinear Schrödinger model. J. Phys. A Math. Theor. 45, 155205 (2012b)

16. Li, J.B.: Exact solution and bifurcations in invariant manifolds for a nonic derivative nonlinear Schrödinger equation. Int. J. Bifurc. Chaos 26, 1650136 (2016)

17. Leta, T.D., Li, J.B.: Bifurcations and exact traveling wave solutions of a generalized derivative of nonlinear Schrödinger equation. Nonlinear Dyn. 85, 1031-1037 (2016)

18. Mirzazadeh, M., Eslami, M., Zerrad, E., Mahmood, M.F., Biswas, A., Belic, M.: Optical solitons in nonlinear directional couplers by sine-cosine function method and Bernoulli's equation approach. Nonlinear Dyn. 81(4), 19331949 (2015)

19. Li, J.B., Chen, F.J.: Exact traveling wave solutions and bifurcations of the dual Ito equation. Nonlinear Dyn. 82, 15371550 (2015)

20. Leta, T.D., Li, J.B.: Exact traveling wave solutions and bifurcations of a further modified Zakharov-Kuznetsov equation. Nonlinear Dyn. 85(4), 2629-2634 (2016)

21. Li, J.B., Chen, G.: Bifurcations of travelling wave solutions for four classes of nonlinear wave equations. Int. J. Bifurc. Chaos 15, 3973-3998 (2005)

22. Li, J.B., Chen, G.: On a class of singular nonlinear traveling wave equations. Int. J. Bifurc. Chaos 17, 4049-4065 (2007)

23. Li, J.B.: Singular Nonlinear Traveling Wave Equations: Bifurcations and Exact Solutions. Science, Beijing (2013)

24. Byrd, P.F., Fridman, M.D.: Handbook of Elliptic Integrals for Engineers and Scientists. Springer, Berlin (1971) 